\title{
Strength of Metals under Vibrations - Dislocation-density-function Dynamics Simulations
}

\author{
B. Cheng, H.S. Leung and A.H.W. Ngan ${ }^{\S}$ \\ Department of Mechanical Engineering, The University of Hong Kong, Pokfulam Road, Hong \\ Kong, P.R. China \\ ${ }^{\S}$ Corresponding author (email: hwngan@hku.hk)
}

\begin{abstract}
It is well-known that ultrasonic vibration can soften metals, and this phenomenon has been widely exploited in industrial applications concerning metal forming and bonding. Recent experiments show that the simultaneous application of oscillatory stresses from audible to ultrasonic frequency ranges can lead to not only softening but also significant dislocation annihilation and subgrain formation in metal samples from the nano- to macro-size range. These findings indicate that the existing understanding of ultrasound softening - that the vibrations either impose additional stress waves to augment the quasi-static applied load, or cause heating of the metal, whereas the metal's intrinsic deformation resistance or mechanism remains unaltered - is far from complete. To understand the softening and the associated enhanced subgrain formation and dislocation annihilation, a new simulator based on the dynamics of dislocation-density functions is employed. This new simulator considers the flux, production and annihilation, as well as the Taylor and elastic interactions between dislocation densities. Softening during vibrations as well as enhanced cell formation are predicted. The simulations reveal the main mechanism for subcell formation under oscillatory loadings to be the enhanced elimination of statistically stored dislocations (SSDs) by the oscillatory stress, leaving behind geometrically necessary dislocations with low Schmid factors which then form the subgrain walls. The oscillatory stress helps the depletion of the SSDs, because the chance for them to meet up and annihilate is increased with reversals of dislocation motions. This is the first simulation effort to successfully predict the cell formation phenomenon under vibratory loadings.
\end{abstract}

Keywords: Dislocation dynamics, dislocation structures, ultrasonics, acoustic softening, dislocation density, subgrain formation 


\section{Introduction}

The phenomenon that the quasi-static stress required for deformation is reduced when ultrasonic vibration is applied [1] has found wide applications in a lot of industrial processes such as ultrasonic welding [2], drilling [3], impact peening [4], and so on. Despite the long history of discovery and application of such acoustoplastic effects [5], there is no universal agreement on the underlying mechanisms. One early explanation proposed is that dislocations can preferentially absorb the acoustic energy and are hence more capable of overcoming their Peierls barriers [1]. The softening effects of the ultrasound are therefore considered the same as those of heating. However, several studies reported the lack of significant temperature rise of the specimen from ultrasound vibration, indicating the peripheral nature of such a thermal effect [68]. Furthermore, Siu, et al. [9] showed that while heating is well-known to harden $\mathrm{Ni}_{3} \mathrm{Al}$, ultrasound irradiation in fact softens this material just as other metals - clearly in $\mathrm{Ni}_{3} \mathrm{Al}$ at least, heating and ultrasound irradiation do not produce equivalent effects on strength.

Several other theories proposed in the past have focused on extrinsic effects of ultrasound. Nevill and Brotzen [10] suggested the stress superposition mechanism, namely, the oscillatory stress reinforces the static stress in a part of the stress cycle, thus lowering the static stress required to maintain the same yielding criteria. Tanibayashi [11] and Malygin [12] proposed different stress-superposition equivalence relationships, based on the assumption that the intrinsic deformation mechanism and microstructure of the material are unaltered by the vibrations. Eaves, et al. [13] postulated that there is a temporary reduction in the coefficient of friction at the contact surface during ultrasonic excitation. However, overwhelming experimental evidences have shown that acoustoplasticity is far from a merely extrinsic effect. First, it is well known that residual softening or hardening may occur in metals after application, and then removal, of ultrasound irradiation [1, 14-16], suggesting that the vibrations can induce permanent changes in the deformation microstructure of the material. This has been recently confirmed by Siu, Ngan and Jones [9], who observed extensive dislocation annihilation and subgrain formation in $\mathrm{Al}, \mathrm{Cu}$ and $\mathrm{Mo}$ samples accompanying ultrasound irradiation in macroindentation experiments. Even in nanoindentation with the vibratory "continuous-stiffnessmeasurement" (CSM) mode switched on, the CSM vibrations can also lead to softening as well as pronounced subgrain formation and lowering of the dislocation density, in $\mathrm{Al}, \mathrm{Cu}$ and $\mathrm{Mo}$ 
when the indentation depth-to-vibration amplitude ratio is smaller than $\sim 100$ to 150 [17]. Since the CSM vibrations are supposed to act as only a probe signal for the measurement of the contact stiffness of the sample, these results show that the CSM nanoindentation technique can become problematic when the vibrational amplitude is not negligible compared with the indent depth. Taking the aforementioned critical ratio of $\sim 100$ to 150 as a general guideline, a typical CSM amplitude of $2 \mathrm{~nm}$ would mean that indents with depths shallower than 200 to $300 \mathrm{~nm}$, or inplane diagonals smaller than $\sim 1.4$ to $2.1 \mu \mathrm{m}$, would be problematic.

The fact that vibratory loadings can lead to extensive dislocation annihilation and subgrain formation [9, 17] is suggestive of certain regulations of the group behaviour of dislocations under such loadings. In addition, dislocation cell formation is also well known to occur during metal fatigue [18], although the eventual fatigue rupture is a consequence of complicated interactions between dislocation plasticity and crack initiation and propagation, which are affected by factors including surface finish and stress concentration. In an attempt to understand dislocation group behavior under oscillatory loading conditions, Siu, Ngan and Jones [9] and Siu and Ngan [19] employed two-dimensional discrete dislocation dynamics (2D-DD) simulations to study the group interactions of dislocations under oscillating and quasi-static stresses. It was found that (i) dislocation annihilation is greatly enhanced under superimposed oscillations leading to softening, due to the fact that during the augmented half cycle of the oscillatory stress, dislocations are made to travel further so that they can explore more environments for annihilation; (ii) in terms of the residual effect of the vibrations, both softening and hardening can occur depending on the residual dislocation quantity, which affects the competition between having sufficient dislocations as carriers of deformation, and dislocation interactions. However, these 2D-DD attempts failed to reproduce subgrain formation, which is an important feature of the deformation microstructure during vibratory loadings. Threedimensional dislocation dynamics (3D-DD) simulations are more realistic than 2D-DD, but a sufficiently large quantity of dislocations has to be involved in order to successfully simulate dislocation patterns, which may also mean impractical computational time. In a typical discrete 3D-DD simulation using parallel computing [20], after a small strain of about $0.02 \%$, a variation in the number of dislocation lines at difference regions was seen. However, dislocation cell formation was far from evident. 
The motivation of this paper is therefore as follows. Instead of using discrete DD to simulate dislocation interactions during the application of oscillatory loadings, we use a dislocation-density-function dynamics approach. The advantage of such an approach over discrete DD is that any quantity of dislocations large or small can be represented by a dislocation density function, and consideration of the time-space evolution of the dislocation density will not be limited by computational power as for discrete DD, especially when the dislocation density is high.

\section{Simulation Details}

A number of authors have modeled the evolution of dislocation density during plastic deformation [21-38], and many of these models involve the crystal kinematics approach in which the evolution of the geometrically necessary dislocations (GNDs) is governed by the change rate of the crystal shape [39]. In doing so, the statistically stored dislocations (SSDs) are ignored. Another common weakness of these models is that the mutual elastic interactions between dislocations are not accounted for, and because the SSDs are not described rigorously, other interactions including Taylor hardening cannot be satisfactorily modeled. Hochrainer and coworkers [40] have proposed a continuum dislocation dynamics (CDD) theory involving a generalized Nye tensor for curved dislocation systems, but numerical application of this theory to realistic plasticity problems remains to be seen.

In this work, we employ a dynamics formulation in which the force on each group of dislocation density is calculated with the Taylor and mutual elastic interactions taken into account. The motion of the dislocation densities is then predicted using a conservative law, with annihilation and generation considered. The coarse-graining concept is assumed valid, which means that representative volume elements (RVE's) exist and can be chosen for the structure to be simulated [41, 42]. The RVE's are similar to the simulation pixels, the size of which should be coarse enough so that the dislocation density inside is sufficiently uniform, but is finer than the microstructure length scale over which the dislocation density typically varies. 


\subsection{The model}

At time $t$, let $\rho_{\alpha}(\vec{r}, t)$ be the density (length per unit volume) of dislocation type $\alpha$ of the cell at position $\vec{r}$. $\alpha$ is specified by the following factors: (i) the slip system $\{\hat{n}, \vec{b}\}$ with $\hat{n}$ being the slip plane unit normal and $\vec{b}$ the Burgers vector, (ii) the dislocation character, which is assumed to be either purely edge $(e)$ or screw $(s)$ following [24, 43, 44], and (iii) the sign of the dislocation (either + or - ). For an fcc single crystal, there are 12 slip systems of the type $\{111\}\left\langle\frac{1}{2}, \frac{\overline{1}}{2}, 0\right\rangle$, and each has 4 dislocation types $e+, e-, s+$ and $s-$, and so there will be 48 types of dislocations to be considered.

The equation governing the evolution of $\rho_{\alpha}(\vec{r}, t)$ is:

$$
\dot{\rho}_{\alpha}(\vec{r}, t)=-\vec{\nabla} \cdot \overrightarrow{\mathrm{J}}_{\alpha}(\vec{r}, t)+\dot{\rho}_{\alpha}^{g e n}+\dot{\rho}_{\alpha}^{a n n}
$$

where $\dot{\rho}_{\alpha}^{g e n}$ and $\dot{\rho}_{\alpha}^{a n n}$ are the generation and annihilation rates respectively. The dislocation flux $\overrightarrow{\mathrm{J}}_{\alpha}(\vec{r}, t)$ here is defined as the length of dislocations of type $\alpha$ moving across a perpendicular surface of unit area per unit time:

$$
\overrightarrow{\mathrm{J}}_{\alpha}(\vec{r}, t)=\rho_{\alpha}(\vec{r}, t) \overrightarrow{\mathrm{v}}_{\alpha}(\vec{r}, t)
$$

The dislocation velocity $\overrightarrow{\mathrm{v}}_{\alpha}(\vec{r}, t)$ here is assumed to be a power law of the effective stress $\tau_{\alpha}^{\text {eff }}$ acting on the dislocation, viz.

$$
\overrightarrow{\mathrm{v}}_{\alpha}(\vec{r}, t)=\operatorname{sgn}\left(\tau_{\alpha}^{\text {eff }}(\vec{r}, t)\right)(\hat{n} \times \hat{\xi}) v_{0}\left|\tau_{\alpha}^{\text {eff }}(\vec{r}, t) / \tau_{0}\right|^{m}
$$

where $v_{0}, \tau_{0}$ and $m$ are material constants, $\operatorname{sgn}(x)$ is the sign function, and $\hat{\xi}$ is the unit vector along the dislocation line corresponding to the dislocation type $\alpha$. The effective glide stress $\tau_{\alpha}^{\text {eff }}$ is

$$
\tau_{\alpha}^{e f f}(\vec{r}, t)=\tau_{\alpha}^{\text {ext }}(\vec{r}, t)-\tau_{\alpha}^{f}-\tau_{\alpha}^{\text {Taylor }}+\tau_{\alpha}^{\text {int }}(\vec{r}, t)
$$

The four terms on the right side of eqn. (4) are, respectively,

(i) the externally applied stress $\tau_{\alpha}^{e x t}$, which is the component of the applied stress on the slip plane (with normal $\hat{n}$ ) along the $\hat{n} \times \hat{\xi}$ direction; 
(ii) the lattice friction $\tau_{\alpha}^{f}$, which is a fixed and small term in the fcc structure;

(iii) the Taylor hardening resistance $\tau_{\alpha}^{\text {Taylor }}=\mu b \sqrt{\sum_{\alpha \prime}\left(\hat{n}_{\alpha} \cdot \hat{\xi}_{\alpha \prime}\right)^{2} \rho_{\alpha \prime}}$ on type $\alpha$ dislocations from the forest network, with the pre-factor taken to be unity for simplicity; and

(iv) the long-range elastic interaction stress $\tau_{\alpha}^{\text {int }}$ from all other dislocations in the system, given as:

$$
\tau_{\alpha}^{i n t}(\vec{r}, t)=\sum_{\alpha^{\prime}, R \leq R_{\text {cutoff }}} \iiint g(\vec{R}, t) \rho_{\alpha^{\prime}}\left(\vec{r}^{\prime}, t\right) d^{3} r^{\prime}
$$

where

$$
\begin{gathered}
g(\vec{R}, t)=-\frac{\mu}{8 \pi}(\hat{n} \times \hat{\xi}) \cdot\left[\left(\vec{b}^{\prime} \times \hat{b}\right) \cdot \vec{\nabla}\left(\nabla^{2} R\right)\right]\left(\hat{\xi} \times \hat{\xi}^{\prime}\right) \\
-\frac{\mu}{8 \pi}(\hat{n} \times \hat{\xi}) \cdot\left\{\left[\vec{b}^{\prime} \times \vec{\nabla}\left(\nabla^{2} R\right)\right] \times \hat{\xi}\right\}\left(\hat{b} \cdot \hat{\xi}^{\prime}\right) \\
-\frac{\mu}{4 \pi(1-v)}(\hat{n} \times \hat{\xi}) \cdot\left[\left(\vec{b}^{\prime} \times \hat{\xi}^{\prime}\right) \cdot \vec{\nabla}\right](\hat{\xi} \times \hat{b} \mathbb{T})
\end{gathered}
$$

In eqn. (5), $\hat{b}=\vec{b} / b, R=\left|\vec{r}-\vec{r}^{\prime}\right|$, and $\vec{\nabla}=\hat{R}(d / d R)$ operates on functions which depend on $R$ alone. The interaction of two dislocation densities is only calculated for those within a cutoff distance $R_{\text {cutoff }}$. Eqn. (5) is a straightforward generalization of the long-range elastic interaction force between two infinitesimally short dislocation segments $d \vec{l}$ and $d \vec{l}^{\prime}$ derived by [Hirth and Lothe [45]]. To obtain the Peach-Koehler glide stress $\tau_{\alpha}^{\text {int }}$ on a unit dislocation segment $\hat{\xi}$, due to all other dislocation densities $\rho_{\alpha^{\prime}}\left(\vec{r}^{\prime}, t\right)$ in the system, we simply set $d \vec{l}=\hat{\xi}$ and $d \vec{l}^{\prime}=$ $\hat{\xi}^{\prime} \rho_{\alpha^{\prime}}\left(\vec{r}^{\prime}, t\right) d^{3} r^{\prime}$ in the Hirth-Lothe equation. The tensor $\mathbb{T}$ in eqn. (5) is given as

$$
\mathbb{T}=\frac{\partial^{2} R}{\partial x_{a} \partial x_{b}} \hat{e}_{a} \otimes \hat{e}_{b}
$$

with $x_{a}$ and $x_{b}$ being the relative coordinates of two interacting dislocation segments. 
Dislocation generation - In the edge-screw idealization used (Fig. 1), dislocation generation arises as a rectangular loop expands (Fig. 1(a)), or as a dislocation line bows out between pinned ends (Fig. 1(b)). The amount of dislocations of type $\alpha$ may therefore change whenever dislocations of another type $\alpha^{\prime}$ on the same slip plane develop velocity gradients along their own line direction, viz.

$$
\dot{\rho}_{\alpha}^{g e n}=\sum_{\alpha \prime \rightarrow \alpha} \partial\left(\rho_{\alpha \prime}, v_{\alpha \prime}\right) / \partial \xi_{\alpha \prime}
$$

As the space is discretized, we take the units for the consideration of dislocation generation to be the cell (pixel) size. Potential dislocation generation is then considered at the walls of neighboring cells. If $(\rho v / \Delta x)^{i} \neq(\rho v / \Delta x)^{i+1}$, then the wall between the $i^{t h}$ and $(i+1)^{t h}$ cells will act as a source of the types shown in Fig. 1, and generation of new dislocation densities will occur. The quantity of new dislocation production at the cell wall thus depends on the difference in $\rho v / \Delta x$ between the two cells, and so eqn. (6) is recast into:

$$
\begin{aligned}
& \dot{\rho}_{e+}^{i, i+1}=F\left[\left(\frac{\rho v}{\Delta x}\right)_{s+}^{i+1}-\left(\frac{\rho v}{\Delta x}\right)_{s+}^{i}\right]+F\left[\left(\frac{\rho v}{\Delta x}\right)_{s-}^{i+1}-\left(\frac{\rho v}{\Delta x}\right)_{s-}^{i}\right] \\
& \dot{\rho}_{e-}^{i, i+1}=F\left[\left(\frac{\rho v}{\Delta x}\right)_{s+}^{i}-\left(\frac{\rho v}{\Delta x}\right)_{s+}^{i+1}\right]+F\left[\left(\frac{\rho v}{\Delta x}\right)_{s-}^{i}-\left(\frac{\rho v}{\Delta x}\right)_{s-}^{i+1}\right]
\end{aligned}
$$

etc.,

where $F(x)$ is the ramp function. However, the quantity of dislocations generated in cell $i$ is limited by the work done by the net effective stress acting on the cell. Since the power density for a given slip system $\alpha$ is $\rho_{\alpha} v_{\alpha} b \tau_{\alpha}^{e f f}$, the generation rates in eqn. (7) are scaled as follows:

$$
\begin{aligned}
& \dot{\rho}_{e+}^{i, i+n} \rightarrow\left(\hat{n}_{w} \cdot \hat{\xi}\right) \frac{\dot{\rho}_{e+}^{i, i+n}}{E_{e}\left(\sum_{n} \dot{\rho}_{e+}^{i, i+n}\right)} \rho_{e+} v_{e+} b \tau_{e+}^{e f f} \\
& \dot{\rho}_{e-}^{i, i+n} \rightarrow\left(\hat{n}_{w} \cdot \hat{\xi}\right) \frac{\dot{\rho}_{e-}^{i, i+n}}{E_{e}\left(\sum_{n} \dot{\rho}_{e-}^{i, i+n}\right)} \rho_{e-} v_{e-} b \tau_{e-}^{e f f} ; \quad \text { etc. },
\end{aligned}
$$

where $(i+n)$ denotes the neighbour cells of $i$, and $E_{e}$ is the line energy per unit length of dislocation. Here, the scaling factors $\rho_{e+} v_{e+} b \tau_{e+}^{e f f} / E_{e}\left(\sum_{n} \dot{\rho}_{e+}^{i, i+n}\right)$, etc., ensure that 
$\rho_{e+} v_{e+} b \tau_{e+}^{e f f}=E_{e}\left(\sum_{n} \dot{\rho}_{e+}^{i, i+n}\right)$, which means that the power density of the effective glide stress on the dislocation population in the cell under consideration is equal to the total line energy, per unit time and volume, of dislocations generated at all the interfaces that this cell makes with its neighbours. The generated densities at the cell wall are partitioned equally into the two cells. The geometrical factor $\left(\hat{n}_{w} \cdot \hat{\xi}\right)$, where $\hat{n}_{w}$ is the cell wall normal, is needed since the dislocation line direction $\hat{\xi}$ would in general not be aligned but inclined with the cell wall.

The generated dislocations should produce a drag effect on the moving dislocation density due to their line tension. However, this drag effect is dropped in the present work, because its incorporation found to produce an unrealistic oscillation effect on the simulation results from time step to time step. The drag effect is a manifestation of the connectivity of the dislocation densities and this is always a weak feature in dislocation-density based models. This aspect should receive further attention in future work.

Density annihilation - Following Arsenlis, M Parks, Becker and V Bulatov [24], annihilation of dislocation densities occurs whenever opposite signed densities come within a critical capture radius. In the edge-screw idealization, the annihilation rates are given as

$$
\begin{gathered}
\dot{\rho}_{e+}^{a n n}=\dot{\rho}_{e-}^{a n n}=-\rho_{e+} \rho_{e-} R_{e}\left|v_{e+}-v_{e-}\right| \\
\dot{\rho}_{s+}^{a n n}=\dot{\rho}_{s-}^{a n n}=-\rho_{s+} \rho_{s-} R_{s}\left|v_{s+}-v_{s-}\right|,
\end{gathered}
$$

where $R_{e}$ and $R_{s}$ are the critical capture radii for edge and screw characters respectively-

Finally, the strain rate is related to the flux by

$$
\dot{\boldsymbol{\varepsilon}}(\vec{r}, t)=\sum_{\alpha}(\vec{b} \otimes \hat{\mathrm{n}})\left[\overrightarrow{\mathrm{J}}_{\alpha}(\vec{r}, t) \cdot(\hat{\mathrm{n}} \times \hat{\xi})\right]
$$

and so the cumulative strain field is obtainable as

$$
\boldsymbol{\varepsilon}(\vec{r}, t)=\int_{0}^{t} \dot{\varepsilon}\left(\vec{r}, t^{\prime}\right) d t^{\prime}
$$

Here, $\boldsymbol{\varepsilon}$ is the engineering strain relative to the original dimensions of the specimen. The Nye tensor, which gives the net GND Burgers vector content threading unit plane [46], is: 


$$
A=\sum_{\alpha}\left(\rho_{\alpha}^{+}-\rho_{\alpha}^{-}\right) \vec{b}_{\alpha} \otimes \vec{\xi}_{\alpha}
$$

\subsection{Numerical implementation}

In this work, we made use of the Finite Volume Method (FVM) to numerically solve eqn. (1). By the first-order "upwind" discretization from basic FVM formulations, the term $-\vec{\nabla}$. $\vec{J}_{\alpha}(\vec{r}, t)$ in eqn. (1) for the $i$-th cell will be

$$
\frac{d \rho_{i}}{d t}+\frac{1}{\Delta x_{i}}\left[(\rho v)_{i+1}-(\rho v)_{i}\right]+\frac{1}{\Delta y_{i}}\left[(\rho v)_{i+1}-(\rho v)_{i}\right]+\frac{1}{\Delta z_{i}}\left[(\rho v)_{i+1}-(\rho v)_{i}\right] .
$$

To handle high gradients, Monotone Upstream-centered Schemes for Conservation Laws (MUSCL) are employed [47].

The temporal evolution was implemented using a forward Verlet integration scheme. The time step $\Delta t$ was set in an adaptive manner via

$$
\Delta t=0.5 \times(\Delta x \Delta y \Delta z)^{\frac{1}{3}} / \max (\text { speed of the densities }),
$$

with also an upper bound of $1.0 \times 10^{-4} \mathrm{~s}$ applied. This adaptive time step can ensure the stability of the time integration scheme, without the overly wasting of computational time when the dislocation velocities are small.

To apply the above model to simulate acoustoplastic effect in a prototypic context, we used a set of material parameters typical in their range as shown in Table 1, rather than the specific properties of a given material. The geometry for the simulations was a rectangular single crystal as shown in Fig. 2, with periodic boundary conditions applied to all three dimensions, which means that as dislocations go out from one side, they return at the opposite side. Thus, effectively, an infinite block was simulated. The three sides of the simulation block were along the $\langle 100\rangle$ directions of the fcc lattice. The simulation block had 50, 50 and 5 simulation cells along $x, y$ and $z$ directions, respectively. This selection was made so as to maximize the amount of information available on the $x-y$ plane. Note that the dimension of the block along $z$ direction is larger than the interaction cutoff distance $\left(R_{\text {cutoff }}\right)$ in the simulations, so dislocations do not interact with their images. 
Initially, the density of each of the 48 types of dislocations at each simulation cell was set randomly from a Gaussian distribution with mean $=\frac{10^{13}}{48} \mathrm{~m}^{-2}$ and variance $=0.25(\text { mean })^{2}$. In this way, the initial total dislocation density of the simulation block is $10^{13} \mathrm{~m}^{-2}$, and the variance in density at different regions is quite small.

For the ease of representation and calculation, the value of external stress used thereafter are normalized by the material shear modulus $\mu$. A simple tensile load was applied along the $x$ direction as shown in Fig. 2, so that all the $\{111\}\left\langle\frac{1}{2}, \frac{1}{2}, 0\right\rangle$ slip systems could be activated. The applied stress consists of two components: (i) a static stress of a certain value, and (ii) an oscillatory stress with a given amplitude at a fixed frequency of $1 \mathrm{kHz}$. This frequency is lower than the typical range of ultrasound. However, it has been experimentally shown that the acoustoplastic effect shows little dependence on the vibration frequency over a wide range from as low as $0.5 \mathrm{~Hz}$ to supersonic $[1,48]$.

Each simulation in the present work typically took 50,000 time steps, which corresponded to about $1.5 \mathrm{~s}$ of real time. The computational time for each simulation was less than one day on a normal personal computer, which represents a major advantage over 3D discrete dislocation dynamic simulations.

\section{Results}

\subsection{Acoustic effect on deformation}

A set of simulations was carried out to see the vibration-induced effects on strength. When a static stress of $2.1 \times 10^{-3} \mu$ is imposed on the specimen in a simulation, the strain gradually accumulates at a decreasing strain rate (black curve in Fig. 3). Simultaneously, the material experiences strain hardening as the dislocation density increases, and the strain rate of the material under a fixed loading condition exhibits an inverse trend with the strain of the material. For other sets of results shown in Fig. 3, a sinusoidal oscillating stress with different amplitudes was superimposed on the static stress, in a time interval $(0.06 s \leq t \leq 0.29 s)$ during the deformation of the specimen. When a small oscillatory loading of $0.25 \times 10^{-3} \mu$ is 
superimposed, the strain rate is barely influenced compared to the static-only case. For vibrations with larger amplitudes, however, the acoustic softening becomes evident and more prominent as the vibration intensity increases. After the removal of the vibrations, residual hardening is observed for larger vibrational amplitudes previously applied, as indicated by a reduction of the strain rate (i.e. slope of the strain-time graph) compared with the moment just before the vibration is switched off.

Using a power law for the strain rate $\dot{\varepsilon} \propto \tau^{m}$, and hence ignoring the possibility that the dislocation density and microstructure can be affected by vibrations, Tanibayashi [11] showed that the reduction $\Delta \tau$ in the static applied stress $\tau_{a}$ due to the application of an oscillatory stress $\tau_{v} \sin (\omega t)$ to achieve the same strain rate without the oscillatory stress is obtainable from:

$$
\left(\tau_{a}-\tau_{i}\right)^{m}=\left\langle\left(\tau_{a}-\tau_{i}-\Delta \tau+\tau_{v} \sin (\omega t)\right)^{m}\right\rangle
$$

where $\langle\ldots\rangle$ denotes time average and $\tau_{i}$ is an internal friction stress. When stress reversal is allowed, the equivalent loading conditions between the oscillatory and static-only cases according to eqn. (14) are specified in Table 2. To test the validity of eqn. (14), a set of simulations was done according to the loading conditions in Table 2, and the results are shown in Fig. 4. It is clear that the strain and strain rate for the simulated cases are different, indicating that Tanibayashi's equivalence principle is incorrect. The breakdown of the Tanibayashi principle illustrates that strain rate does not obey a simple law $\dot{\varepsilon} \propto \tau^{m}$, and since $\dot{\varepsilon} \sim \rho b v$, the dislocation density and microstructure must be modified by the vibrations.

\subsection{Vibration effects on microstructure}

To further explore the acoustoplastic effect on the internal structure of the material, we examined the evolution history of two selected cases: (i) static loading of $2.1 \times 10^{-3} \mu$ only, and (ii) oscillatory loading of $0.98 \times 10^{-3} \mu+\sin (2000 \pi t) \times 1.89 \times 10^{-3} \mu$ at a fixed frequency of $1 \mathrm{kHz}$. The strain response of the two cases is illustrated in Fig. 4. In Fig. 5(a), the evolution of the total dislocation density is plotted. While the dislocation density for the oscillatory case is much higher at the end of the simulation, it stagnates for the first $0.02 \mathrm{~s}$. The change in the total dislocation density of the whole specimen depends on generation and annihilation, and in Fig. 
5(b), the rates of generation and annihilation are plotted separately. Due to the oscillating stress, the generation and annihilation rates in the vibratory case fluctuate throughout the simulation, and shortly after the simulation starts $(0.01 s \leq t \leq 0.02 s)$, both generation and annihilation become highly active but they roughly cancel out each other, thus explaining why the overall dislocation density stagnates for the first $0.02 \mathrm{~s}$. At around $0.03 \mathrm{~s}$, the annihilation rate undergoes a sharp drop, and although the generation rate also declines at the same time, the decay is much slower. On the contrary, for the static-only case, both generation and annihilation are slower than the oscillatory case on average, and generation in general is faster than annihilation. Therefore, the overall dislocation density keeps rising steadily.

Fig. 6 shows the evolution of the dislocation distribution over an $x-y$ section of the specimen for both cases. The initial density distribution shows a small variation. For the staticonly case in Fig. 6(a), the dislocation density in some regions builds up rapidly, while that in other regions stagnates or even declines as time proceeds. The accumulation of dislocation density and the formation of patterns are a gradual process starting from the onset of the simulation. At the end of the $1.5 \mathrm{~s}$, regions with either very high or very low dislocation densities have developed, with the highest dislocation density reaching $10^{15} \mathrm{~m}^{-2}$, while the lowest values remaining below $10^{13} \mathrm{~m}^{-2}$. The areas with high and low densities juxtapose and form alternating patterns. The regions with high dislocation density appear in the form of disconnected speckles. For the oscillatory case in Fig. 6(b), the dislocation density remains low in the initial stage, but pattern formation appears rather quickly around $t=0.04 \mathrm{~s}$. The final dislocation density distribution at the end of the simulation also has high and low density regions. However, in contrast with the disconnected speckle pattern for the static loading case, the distribution plot of the oscillatory case consists of low-density regions surrounded by long stripes of high density, which closely resemble a subcell structure. The size of the subcells is on the order of a micron.

The presence of cell formation can be further revealed by probability density distributions of dislocation densities. A dislocation-cell structure is represented by two peaks in the probability distribution function of the dislocation density, with one peak at a higher density corresponding to the cell walls, and the second peak at a much lower density corresponding to the cell interiors. The temporal evolution of the probability density distribution of the dislocation density is shown in Fig. 7. Since the total dislocation density for the static and oscillatory cases 
are quite different at the same time instant, the probability distributions in Fig. 7(a-c) are compared at various total densities for the static-only and oscillatory cases. The total density increased to $2 \times 10^{13} \mathrm{~m}^{-2}$ for the static-only case at $t=0.008 \mathrm{~s}$, and at $t=0.017 \mathrm{~s}$ for the oscillatory case (Fig. 7(a)). For the static loading case, the dislocation density distribution exhibits a dual-peak feature, with the peak at higher density corresponding to the high density dots in Fig. 6. The dislocation density distribution for the oscillatory case has a single sharp peak, which corresponds to the nearly uniform distribution in Fig. 6. The uniform dislocation density is presumably the result of the balance between the dislocation generation and quick annihilation at the time. The total density reached $5 \times 10^{13} \mathrm{~m}^{-2}$ for the static-only case at $t=0.080 \mathrm{~s}$, and at $t=$ 0.072s for the oscillatory case (Fig. 7(b)). Between 0.008s and 0.08s, the dual-peak feature for the static loading case quickly turned into a single peak. For the oscillatory case, a dual-peak feature emerged with the two modal dislocation densities differ by about five times, in accordance with the shutting down of the high annihilation mode in Fig. 5(b). The total density reached $6 \times 10^{13} \mathrm{~m}^{-2}$ for the static-only case at $t=0.716 \mathrm{~s}$, and at $t=0.086 \mathrm{~s}$ for the oscillatory case (Fig. 7(c)). The dual-peak feature in the dislocation density probability distribution for the oscillatory case is even more pronounced at the total density of $6 \times 10^{13} \mathrm{~m}^{-2}$, with the two modal dislocation densities differ by about ten times. After that, the low-density region for the oscillatory loading case underwent a moderate increase in density, so that the separation between the two peaks gradually became smaller towards the end of the simulation at $t=1.5 \mathrm{~s}$ (Fig. 7(d)), but the bimodal distribution of the dislocation density is still very clear.

To further provide information on cell formation, Nye tensors are calculated according to eqn. (12) and are plotted in Fig. 8 for the structures at the end of the simulation at $t=1.5 \mathrm{~s}$. Here, the nine $A_{i j}$ components of the Nye tensor are specified by the label $(i, j)$, where $i$ is along the GND Burgers vector component, and $j$ indicates normal direction of the unit plane the GNDs are threading. For the static-only case in Fig. 8(a), the plots for the Nye tensor components at the end of the simulation exhibit small and discrete patches dispersing rather evenly throughout the specimen. This indicates that GNDs are rather randomly dispersed in space without organizing into a subcell structure, and this is consistent with the dislocation density map in Fig. 6(a) at $t=$ $1.5 \mathrm{~s}$. On the contrary, for the oscillatory case in Fig. 8(b), a number of Nye tensor components, in particular the $(2,3),(3,1)$ and $(3,2)$ components, show distinctive long stripes which tend to 
align along the $x$ and $y$ directions of the simulation domain, indicating that GNDs form wall-like structures along these directions. The GND walls are also separated by about a micron, and their locations roughly coincide with the long stripes of high dislocation densities in Fig. 6(b) at $t=$ 1.5 s. The Burgers vector components for these Nye tensor components of $(2,3),(3,1)$ and $(3,2)$ are along the $y$ or $z$ direction which are perpendicular to the tensile axis (the $x$ direction). The associated GNDs would therefore have low Schmid factors and this explains why they remain rather static in the structure despite the oscillatory loading. The Nye tensor plots in Fig. 8(b) therefore confirm that the long stripes with high dislocation densities seen in Fig. 6(b) at the end of the simulation (at $1.5 \mathrm{~s}$ ) are mainly composed of GNDs with low Schmid factors. The cell-like structures in Fig. 6(b) at $1.5 \mathrm{~s}$ should therefore be subgrains which are misoriented with respect to each other.

\subsection{Cell size and initial dislocation density}

It has been suggested, from a principle of similitude, that the subgrain size should be inversely proportional to the square root of the initial dislocation density $[49,50]$. For specimens under oscillatory loadings, cell formation is prominent even when the overall strain has reached just a few percent [9]. To investigate how the subgrain size varies with the initial dislocation density, a set of simulations was performed for three specimens with different initial dislocation densities $\left(10^{13} \mathrm{~m}^{-2}, 4 \times 10^{13} \mathrm{~m}^{-2}\right.$ or $\left.9 \times 10^{13} \mathrm{~m}^{-2}\right)$ under vibrational loadings. The loading applied was scaled according to the initial strength corresponding to the different initial dislocation density, but the ratio between the amplitude of the vibrational stress and the static stress was always maintained at $1.89 \times 10^{-3} \mu / 0.98 \times 10^{-3} \mu$. The final equilibrium strains for the three cases are about a few percent. When the strain reached 3\%, snapshots of the dislocation distributions are shown in Fig. 9. For cases (a) and (b) with initial dislocation density of $10^{13} \mathrm{~m}^{-2}$ and $4 \times 10^{13} \mathrm{~m}^{-2}$ respectively, the formation of dislocation cellular structure is quite obvious and regular. In Fig. 9 (a,b) the cell walls are indicated by red dash lines and the cells themselves are numbered. The cell size can be determined by an averaging method, namely, the average cross-sectional area of the cells is the simulation region divided by the number of cells it contains, and then the subcell size is estimated as the square root of cellular cross-sectional area. From Fig. 9, the ratio between subcell size in case (a) and (b) is about 1.3. This ratio is lower 
than the value predicted from the inverse-square-root relationship between cell size and initial dislocation density [49], which should be 2. However, an inverse relationship between the cell size and the dislocation density is evident. With the initial density as high as $9 \times 10^{13} \mathrm{~m}^{-2}$, the high density regions do not appear similar to regular cell walls, but are clearly different from the disconnected speckle pattern in Fig.6(a).

\section{Discussion}

From the simulation results, acoustic softening was found to become more prominent at higher vibration amplitudes, once the oscillation exceeds a threshold. This is consistent with experimental findings - Langenecker [1], Kozlov and Selitser [48], Ohgaku and Takeuchi [51] and many others all found more prominent softening as the ultrasonic intensity increases. Furthermore, Fig. 3 shows that after the vibrations are switched off, a small residual hardening effect, indicated by a smaller strain rate, arises when the previous vibrational amplitude is large. Residual hardening has also been found experimentally especially after the application of sufficiently large vibration intensities [16]. Tanibayashi's equivalence principle (eqn. 14) was found to be invalid, since the dislocation density and microstructure changes during the deformation are significant, which contradicts the assumption in the equivalence consideration. We have also examined the final dislocation distribution of the specimen, and found much enhanced dislocation cell formation under oscillatory loading. The Nye tensor plots in Fig. 9(b) reveal distinctive GND walls with spacing of about a micron, which imply the emergence of lattice rotations characteristic of a subgrain microstructure. Siu, Ngan and Jones [9] and [Siu and Ngan [52]][Siu and Ngan [52]][Siu and Ngan [52]][Siu and Ngan [52]][Siu and Ngan [52]]Siu and Ngan [52] have experimentally confirmed enhanced subgrain formation under ultrasound irradiation and under CSM nanoindentation in different metals. In both simulations and experiments, the subgrains formed are on the order of a micron large, consistent with the present simulation results. To the best of our knowledge, this is the first successful attempt to be able to predict dislocation patterning resembling subgrain formation under vibrational loadings.

We further investigated the underlying mechanism for the dislocation cell formation. The dislocation-density evolution in Fig. 5 and 6 illustrates that under vibrational loading, there exists 
a plateau region at the initial stage of the simulation, where the overall dislocation density remains low and pattern formation is hindered. Together with the dislocation generation and annihilation rates plotted in Fig. 5(b), it is clear that the plateau region is associated with a particularly high annihilation rate at the beginning. When the rapid annihilation mode is shut down, the regional and overall densities begin to rise steadily. A question to ask is therefore: what inactivates the high annihilation rate? From eqn. (9), the annihilation rate is mainly governed by two factors: the dislocation velocity and the number of dislocation dipoles near a simulation cell. On a slip system near a simulation pixel, if there is an abundance of one type (e.g. $e+)$, but a depletion of the opposite type (e.g. $e-$ ), the annihilation rate will be quite low. In other words, the polarization of dislocations hinders the annihilation process. To quantitatively represent the dislocation polarization $(P)$, we make use of the Nye tensor (eqn. 12):

$$
P=\sqrt{\sum A_{i j}^{2}}
$$

In Fig. 10 the evolution of dislocation polarization with respect to time is shown for the staticonly and vibratory case. The polarity under static loading rises continuously from the beginning of the simulation, while under oscillatory loading, it stalls for the first $0.02 \mathrm{~s}$, and the starting point of polarity rise coincides the shutting down of the high annihilation mode in Fig. 5(b). It is therefore evident that the vibrational loading promotes the exhaustion of dipole dislocations, or SSDs, in the initial dislocation structure. The depletion of dipoles and the intensified dislocating polarization in turn impede the annihilation of dislocations. The high annihilation mode under vibrational loading soon shuts down, resulting in the rapid increase of total and local dislocation density in the specimen. The final Nye tensor plots in Fig. 8 further support the fact that under oscillatory loading, the high annihilation event quickly exhausts a large part of the SSDs in the simulation cells, leaving behind patches of GNDs with low Schmid factors which correspond to a large dislocation polarity. The presence of GNDs in a certain volume is closely related to lattice misorientation, which is the basic feature of subgrain formation.

The quicker depletion of the SSDs under oscillatory loadings is due to the reversal of dislocation motion, which increases the chance for SSDs to meet up and annihilate. This phenomenon was also seen in discrete DD simulations, although the dislocation quantity that can be handled by discrete DD did not allow subgrain formation to be predicted [19]. The ease of 
cross slip is likely a factor affecting the annihilation of SSDs as suggested by DD simulations [19], but even in the present simulations in which cross slip is suppressed, subcell structures are seen with vibrations on. This indicates that even in materials with low stacking fault energy (SFE), subcell structures can follow oscillatory loadings. In fact, experiments have indicated that subgrain formation is prominent both in $\mathrm{Al}$ and $\mathrm{Cu}$ following deformation augmented by ultrasonic vibrations $[9,52]$ - the fact that $\mathrm{Al}$ and $\mathrm{Cu}$ have very different SFE indicates that cross slip is not an overriding factor for subgrain formation, although fine details of the subgrains and the speed at which they are formed may be influenced by the ease of cross slip. Further work focusing on the role of cross slip within the present model framework is worthwhile.

A further point to address is how the dislocation cell formation corresponds to the softening under vibrational loading. Fig. 11 shows the overall components of the material's strength under static-only and oscillatory loadings. It can be seen that the average elastic interaction stress between dislocations $\left(\overline{\left|\tau_{\alpha}^{i n t}\right|}\right)$ stress under oscillatory loading is larger than that under static loading, but the normalized Taylor hardening resistance $\overline{\left|\tau_{\alpha}^{\text {Taylor }}\right|} / \sqrt{\rho^{\text {total }}}$ in two cases is similar. Since the magnitude of the interaction stress $\tau_{\alpha}^{\text {int }}$ is only about $5 \%$ of the Taylor interaction stress $\tau_{\alpha}^{\text {Taylor }}$, the different strength components given in Fig. 11 do not reveal a clear relation between subcell formation and softening with vibration on. This is likely due to the fact that, although the overall Taylor resistance is not lowered under the vibration, the local resistance may be significantly lowered in subgrain interiors with much lower dislocation densities. To verify this, two sets of simulation were done under the following conditions: (a) oscillatory loading of $0.98 \times 10^{-3} \mu+\sin (2000 \pi t) \times 1.89 \times 10^{-3} \mu$ were applied for $0.086 \mathrm{~s}$ until the dislocation density reached $6 \times 10^{13} \mathrm{~m}^{-2}$, followed by applying static loading of $2.1 \times 10^{-3} \mu$, and (b) static loading of $2.1 \times 10^{-3} \mu$ all along. When the total dislocation density for case (a) has reached $6 \times 10^{13} \mathrm{~m}^{-2}$, the dislocation cell formation is prominient. In contrary, the cell structure for case (b) is absent throughout. After the density has reached $6 \times 10^{13} \mathrm{~m}^{-2}$, the subsequent strain responses for the two cases under static loading at $2.1 \times 10^{-3}$ are shown in Fig. 12. Despite the same initial dislocation density, the strain rate of the specimen pre-treated with vibration is about 10 times higher than its counterpart which is always under static loading. These results therefore show that with cellular structure formation, the material is softer compared to the case of no cellular structure formation at the same total dislocation density. 
Finally, it is useful to comment on the issue of numerical convergence in the present simulations. To check for convergence, we altered the dimensions of the simulation block as well as those of the simulation pixels. As mentioned above, the original size of the simulation block was 50 pixels along $x, 50$ pixels along $y$, and 5 pixels along $z$, with each pixel being a cube with length $200 b$. Further simulations were performed using a simulation block size with a doubled length along $z$, i.e. 10 pixels, and similar dislocation-density structures as the original block size with and without oscillatory loading as in Fig. 6 were observed. This shows that the results presented so far are convergent with respect to the size of the simulation block. When the size of the simulation pixel was doubled or halved, similar results as the original pixel size were also obtained for the case of static loading. Under oscillatory loadings, subcell dislocation structures were also obtained with the pixel size doubled or halved, but the subcell size was found to increase with the pixel size. As an example, under an oscillatory stress of $0.98 \times 10^{-3} \mu+$ $\sin (2000 \pi t) \times 1.89 \times 10^{-3} \mu$ for $0.3 \mathrm{~s}$, the resultant subcell size was found to be close to $\sim 25$ pixels irrespective of their actual size, i.e. when the pixel size is $100 b$, the subcell size is close to $\sim 2500 \mathrm{~b}$, and when the pixel size is $200 \mathrm{~b}$, the subcell size is close to $\sim 5000 \mathrm{~b}$, and so on, and such a trend is independent of the size of the entire simulation block. As discussed above, the formation of the subcells is due to the enhanced elimination of the SSDs by the oscillatory stress, while GNDs with low Schmid factors are left behind to form the subcell walls. Thus, the length of around 25 pixels here represents the wavelength over which clearance of the SSDs is most effective. It should be noted that, by virtue of the edge-screw idealization shown in Fig. 1 and equations such as eqn. (7), the pixel size is an explicit parameter in the present model, and so an observed scaling of the microstructural pattern size with the pixel size should not be surprising. Such a scaling should also not be seen as a disturbing feature of the model because, in the coarse-graining concept, the pixel or RVE size is a meso-scale property which cannot be indefinitely shrunk [41,42]. As said before, the pixel size has to be small enough compared to the length scale of the microstructure to be modeled, but large enough so that the dislocation structure inside is sufficiently featureless. Therefore, a change in the pixel or RVE size corresponds to a change in the interaction details of the dislocations, and may give rise to different predictions. Alternatively, the size of the RVE represents as a mesoscopic material parameter which may be obtained by fitting the simulated results to experimental observations. 


\section{Conclusions}

Simulations based on dislocation-density dynamics show that acoustic softening becomes more prominent at higher vibrational intensities. The reason is far from a simple added-stress effect, in which the oscillatory loading adds to the static loading to make the dislocations travel faster. Instead, the oscillatory loading promotes dipole annihilation, resulting in enhanced polarity and therefore strong GND patterns, resembling cell structure formation. The well-known softening effect of oscillatory loading is therefore mainly due to the enhanced cell formation. The simulation results tally well with experimental observations of strong dislocation pattern formation under vibrational loadings.

\section{Acknowledgment}

This work is supported by funding from Kingboard Endowed Professorship in Materials Engineering.

\section{References}

[1] B. Langenecker, Sonics and Ultrasonics, IEEE Transactions on 13 (1966) p. 1.

[2] J. Tsujino, M. Hongoh, R. Tanaka, R. Onoguchi and T. Ueoka, Ultrasonics 40 (2002) p. 375.

[3] R. Neugebauer and A. Stoll, Journal of materials processing technology 149 (2004) p. 633.

[4] B.N. Mordyuk and G.I. Prokopenko, Journal of Sound and Vibration 308 (2007) p. 855.

[5] F. Blaha and B. Langenecker, Naturwissenschaften 42 (1955) p. 0.

[6] Y. Daud, M. Lucas and Z. Huang, Journal of materials processing technology 186 (2007) p. 179.

[7] Y. Daud, M. Lucas and Z. Huang, Ultrasonics 44 (2006) p. e511.

[8] Z. Huang, M. Lucas and M.J. Adams, Ultrasonics 40 (2002) p. 43.

[9] K.W. Siu, A.H.W. Ngan and I. Jones, International Journal of Plasticity 27 (2011) p. 788.

[10] G. Nevill and F.R. Brotzen, Proceeding-American Society for Testing Material 57 (1957) p. 751.

[11] M. Tanibayashi, physica status solidi (a) 128 (1991) p. 83.

[12] G. Malygin, Physics of the Solid State 42 (2000) p. 72.

[13] A. Eaves, A. Smith, W. Waterhouse and D. Sansome, Ultrasonics 13 (1975) p. 162. 
[14] H. Huang, A. Pequegnat, B. Chang, M. Mayer, D. Du and Y. Zhou, Journal of Applied Physics 106 (2009) p. 113514.

[15] I. Lum, H. Huang, B. Chang, M. Mayer, D. Du and Y. Zhou, Journal of Applied Physics 105 (2009) p. 024905.

[16] N. Tyapunina, V. Blagoveshchenskii, G. Zinenkova and Y.A. Ivashkin, Soviet Physics Journal 25 (1982) p. 569.

[17] K.W. Siu and A.H.W. Ngan, Philosophical Magazine 93 (2013) p. 449.

[18] D. Kuhlmann-Wilsdorf and C. Laird, Materials Science and Engineering 46 (1980) p. 209.

[19] K.W. Siu and A.H.W. Ngan, Philosophical Magazine 91 (2011) p. 4367.

[20] M. Verdier, M. Fivel and I. Groma, Modelling and Simulation in Materials Science and Engineering 6 (1998) p. 755.

[21] A. Acharya, Journal of the Mechanics and Physics of Solids 49 (2001) p. 761.

[22] R. Aghababaei and S.P. Joshi, International Journal of Plasticity (2012).

[23] A. Alankar, P. Eisenlohr and D. Raabe, Acta Materialia 59 (2011) p. 7003.

[24] A. Arsenlis, D. M Parks, R. Becker and V. V Bulatov, Journal of the Mechanics and Physics of Solids 52 (2004) p. 1213.

[25] S. Bargmann, B. Svendsen and M. Ekh, Computational Mechanics 48 (2011) p. 631.

[26] P. Engels, A. Ma and A. Hartmaier, International Journal of Plasticity 38 (2012) p. 159.

[27] L. Evers, W. Brekelmans and M. Geers, Journal of the Mechanics and Physics of Solids 52 (2004) p. 2379.

[28] I. Groma, Physical Review B 56 (1997) p. 5807.

[29] C. Hirschberger, R. Peerlings, W. Brekelmans and M. Geers, Modelling and Simulation in Materials Science and Engineering 19 (2011) p. 085002.

[30] D. Li, H. Zbib, X. Sun and M. Khaleel, International Journal of Plasticity (2013).

[31] Z. Liu, Z. Zhuang, X. Liu, X. Zhao and Z. Zhang, International Journal of Plasticity 27 (2011) p. 201.

[32] A. Ma, F. Roters and D. Raabe, Acta Materialia 54 (2006) p. 2169.

[33] J. Pontes, D. Walgraef and E. Aifantis, International journal of plasticity 22 (2006) p. 1486.

[34] S. Puri, A. Das and A. Acharya, Journal of the Mechanics and Physics of Solids 59 (2011) p. 2400.

[35] D. Walgraef and E.C. Aifantis, Journal of applied physics 58 (1985) p. 688.

[36] I. Watanabe, D. Setoyama, N. Iwata and K. Nakanishi, International Journal of Plasticity 26 (2010) p. 570.

[37] S. Yefimov and E. Van der Giessen, International Journal of Solids and Structures 42 (2005) p. 3375.

[38] Z. Zhou and Y. Sun, Chinese Science Bulletin 49 (2004) p. 1527.

[39] R.J. Asaro and J. Rice, Journal of the Mechanics and Physics of Solids 25 (1977) p. 309.

[40] T. Hochrainer, M. Zaiser and P. Gumbsch, Philosophical Magazine 87 (2007) p. 1261.

[41] W. Drugan and J. Willis, Journal of the Mechanics and Physics of Solids 44 (1996) p.

497.

[42] T. Kanit, S. Forest, I. Galliet, V. Mounoury and D. Jeulin, International Journal of Solids and Structures 40 (2003) p. 3647.

[43] E. Busso, F. Meissonnier and N. O'dowd, Journal of the Mechanics and Physics of Solids 48 (2000) p. 2333.

[44] A. Alankar, D.P. Field and H.M. Zbib, Philosophical Magazine 92 (2012) p. 3084. 
[45] J. Hirth and J. Lothe, Theory of dislocations, Krieger, Melbourne, FL, 1992, p. 125.

[46] J.F. Nye, Physical Properties of Cristals: Their Representation by Tensors and Matrices, Oxford University Press, 1985.

[47] P. Wesseling, Principles of computational fluid dynamics, Springer, 2001, p. 381.

[48] A. Kozlov and S. Selitser, Materials Science and Engineering: A 102 (1988) p. 143.

[49] D.L. Holt, Journal of Applied Physics 41 (1970) p. 3197.

[50] L.E. Murr and D. Kuhlmann-Wilsdorf, Acta Metallurgica 26 (1978) p. 847.

[51] T. Ohgaku and N. Takeuchi, physica status solidi (a) 111 (1989) p. 165.

[52] K.W. Siu and A.H.W. Ngan, Materials Science and Engineering: A (2013). 
Table 1 - Simulation parameters

\begin{tabular}{|l|c|}
\hline \multicolumn{1}{|c|}{ Parameters } & Values \\
\hline Burgers vector $b$ & $2.863 \times 10^{-10} \mathrm{~m}$ \\
\hline Material shear modulus $\mu$ & $26 \times 10^{9} \mathrm{~Pa}$ \\
\hline Poisson's ratio & 0.3 \\
\hline Stress exponent $m$ for dislocation velocity, eqn. (3) & $1 \mathrm{~ms}^{-1}$ \\
\hline$v_{0}$ for dislocation velocity, eqn. (3) & $7.8 \times 10^{6} \mathrm{~Pa}$ \\
\hline$\tau_{0}$ for dislocation velocity, eqn. (3) & $0.77 \times 10^{-4} \mu$ \\
\hline Lattice friction $\tau_{\alpha}^{f}$, eqn. (4) & $50(x) \times 50(y) \times 5(z)$ cells \\
\hline $\begin{array}{l}\text { Crystal dimensions (with periodic boundary } \\
\text { conditions), Fig. 2 }\end{array}$ & $200 b, 200 b, 200 b$ \\
\hline Cell size $\Delta x, \Delta y, \Delta z$ section 2.2 & $400 b$ \\
\hline Cutoff distance for interaction $R_{\text {cutoff }}$, eqn. (5) & $200 b$ \\
\hline Capture radius for annihilation of edge $R_{e}$, eqn. (9) & $200 b$ \\
\hline Capture radius for annihilation of screw $R_{s}$, eqn. (9) & \\
\hline
\end{tabular}

Table 2 - The equivalent static stress and oscillation amplitude for $m=5$ according to eqn. (14)

\begin{tabular}{|c|c|}
\hline Static stress $\left(\tau_{a}-\Delta \tau\right)$ & Amplitude of oscillation $\left(\tau_{v}\right)$ \\
\hline $2.1 \times 10^{-3} \mu$ & 0 \\
\hline $1.92 \times 10^{-3} \mu$ & $0.63 \times 10^{-3} \mu$ \\
\hline $1.49 \times 10^{-3} \mu$ & $1.26 \times 10^{-3} \mu$ \\
\hline $0.98 \times 10^{-3} \mu$ & $1.89 \times 10^{-3} \mu$ \\
\hline
\end{tabular}




\section{Figure Captions}

Figure 1 - Production of new dislocations in the edge-screw idealization by (a) expansion of a rectangular loop, (b) bow-out.

Figure 2 - Specimen for simulation.

Figure 3 - Strain response of materials under oscillation of different amplitudes from 0.06-0.29s.

Figure 4 - Strain response of materials under different loading conditions.

Figure 5 - (a) Overall dislocation density evolution during the simulations. (b) The dislocation generation and annihilation rate during the first $0.15 \mathrm{~s}$.

Figure 6 - Dislocation density $\left(\mathrm{m}^{-2}\right)$ evolution at different time points for (a) static loading of $2.1 \times 10^{-3} \mu$, (b) oscillatory loading of $0.98 \times 10^{-3} \mu+\sin (2000 \pi t) \times 1.89 \times 10^{-3} \mu$. Time is specified under each snapshot.

Figure 7 - Probability density functions for dislocation densities when the total dislocation densities under the two different loading conditions are both at (a) $2 \times 10^{13} \mathrm{~m}^{-2}$, (b) $5 \times$ $10^{13} \mathrm{~m}^{-2}$, and (c) $6 \times 10^{13} \mathrm{~m}^{-2}$. (d) shows the distribution at the end of the simulations. The abscissa is the $\log _{10}$ value of the total dislocation density, and the vertical scale denotes the occurrence frequency of a particular density in the simulation block.

Figure 8 - Nye's tensor plot at the end of the simulations, in units of $\mathrm{m}^{-1}$. The label $(\mathrm{i}, \mathrm{j})$ denotes the $A_{i j}$ component of the tensor. (a) Static loading of $2.1 \times 10^{-3} \mu$ only, (b) oscillatory loading of $0.98 \times 10^{-3} \mu+\sin (2000 \pi t) \times 1.89 \times 10^{-3} \mu$.

Figure 9 - Dislocation density distribution for specimens with different initial densities at strain $=3 \%$. The current total dislocation density is specified under each snapshot. (a) Specimen with initial density $10^{13} \mathrm{~m}^{2}$ under oscillatory loading of $0.98 \times 10^{-3} \mu+\sin (2000 \pi t) \times 1.89 \times$ $10^{-3} \mu$. (b) Specimen with initial density $4 \times 10^{13} \mathrm{~m}^{2}$ under oscillatory loading of $1.25 \times 10^{-3} \mu$ $+\sin (2000 \pi t) \times 2.41 \times 10^{-3} \mu$. (c) Specimen with initial density $9 \times 10^{13} \mathrm{~m}^{2}$ under oscillatory loading of $1.69 \times 10^{-3} \mu+\sin (2000 \pi t) \times 3.26 \times 10^{-3} \mu$. Red dash lines indicate the position of cell walls, and different cells are numbered.

Figure 10- Dislocation polarity evolution for the two cases in simulations. 
Figure 11 - The evolution of elastic interaction stress between dislocations $\overline{\left|\tau_{\alpha}^{i n t}\right|}$, and normalized Taylor hardening resistance $\overline{\left|\tau_{\alpha}^{\text {Taylor }}\right|} / \sqrt{\rho^{\text {total }}}$ for specimens under two different loading conditions.

Figure 12 - The strain response of two specimens under the static loading of $2.1 \times 10^{-3} \mu$. Black curve: the specimen was pretreated with oscillatory loading of $0.98 \times 10^{-3} \mu+\sin (2000 \pi t) \times$ $1.89 \times 10^{-3} \mu$ for $0.086 \mathrm{~s}$ until the dislocation density reached $6 \times 10^{13} \mathrm{~m}^{-2}$. Red curve: the specimen was pretreated with static loading of $2.1 \times 10^{-3} \mu$ only, until the dislocation density reached $6 \times 10^{13} \mathrm{~m}^{-2}$. 


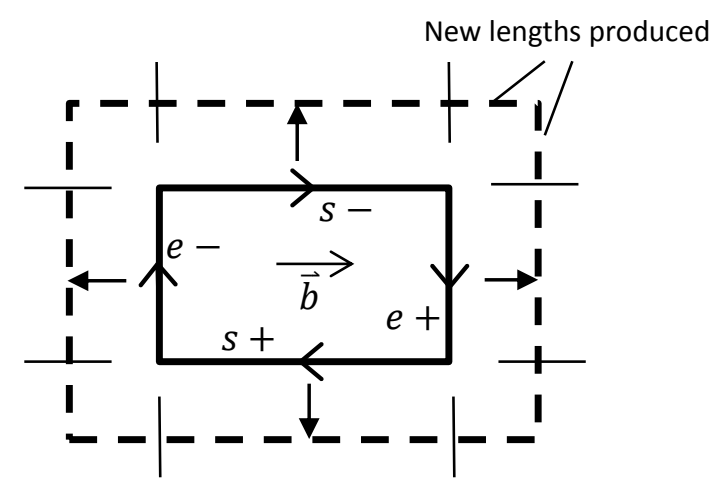

(a)

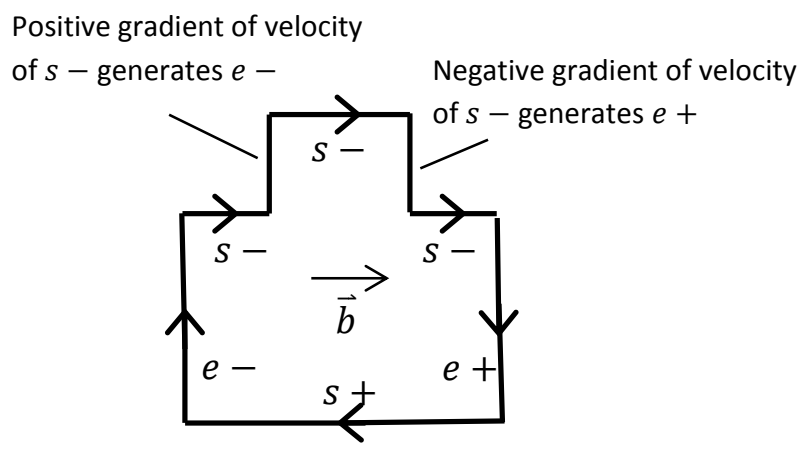

(b)

Figure 1 - Production of new dislocations in the edge-screw idealization by (a) expansion of a rectangular loop, (b) bow-out of a dislocation segment. 


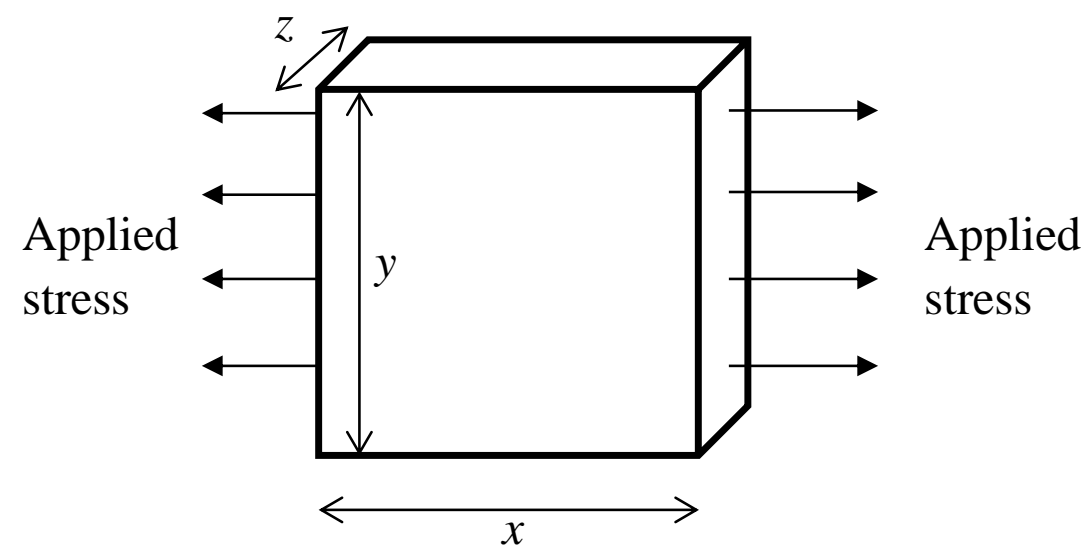

Figure 2 - Specimen for simulation. 


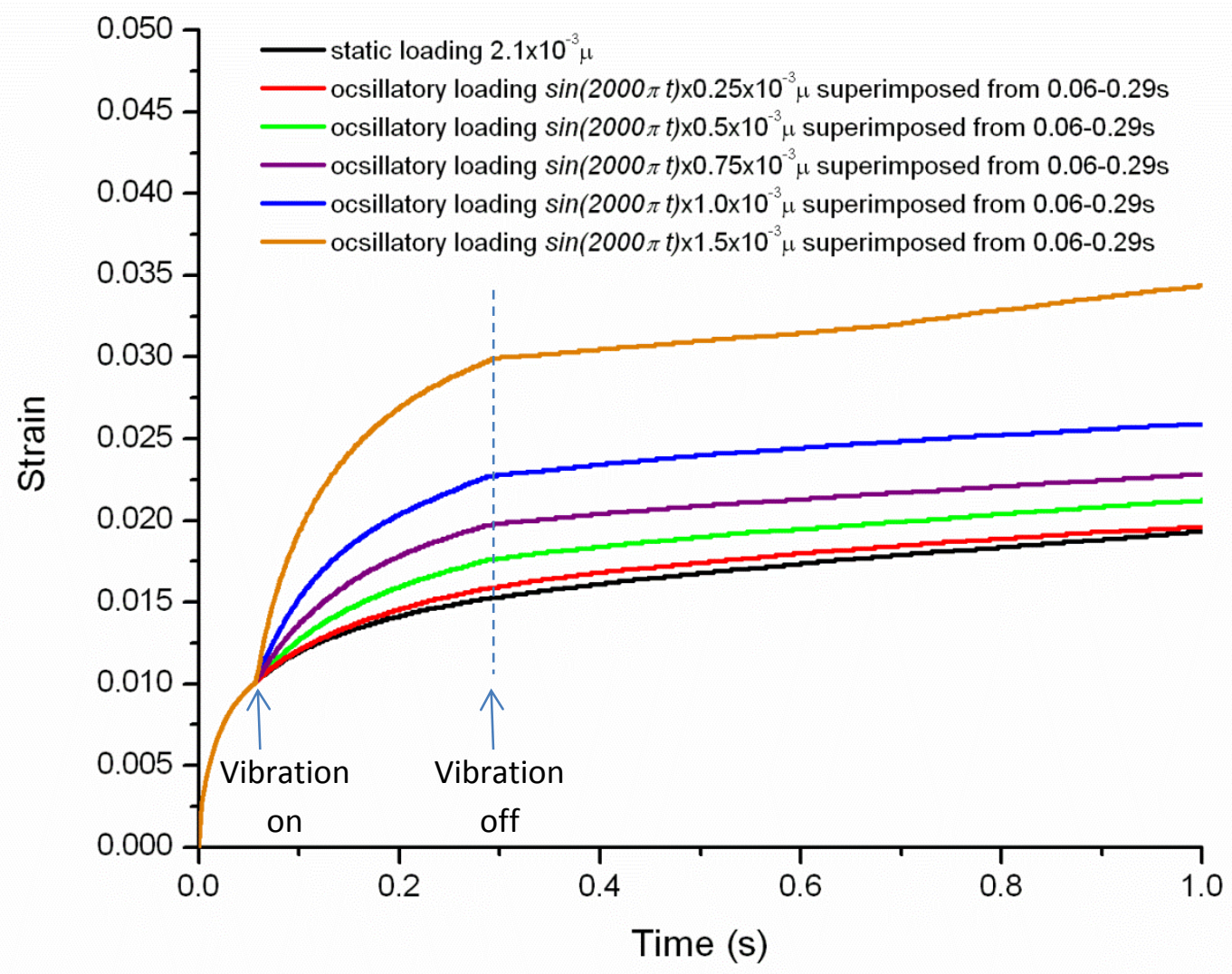

Figure 3 - Strain response of materials under oscillation of different amplitudes from 0.06-0.29s. 


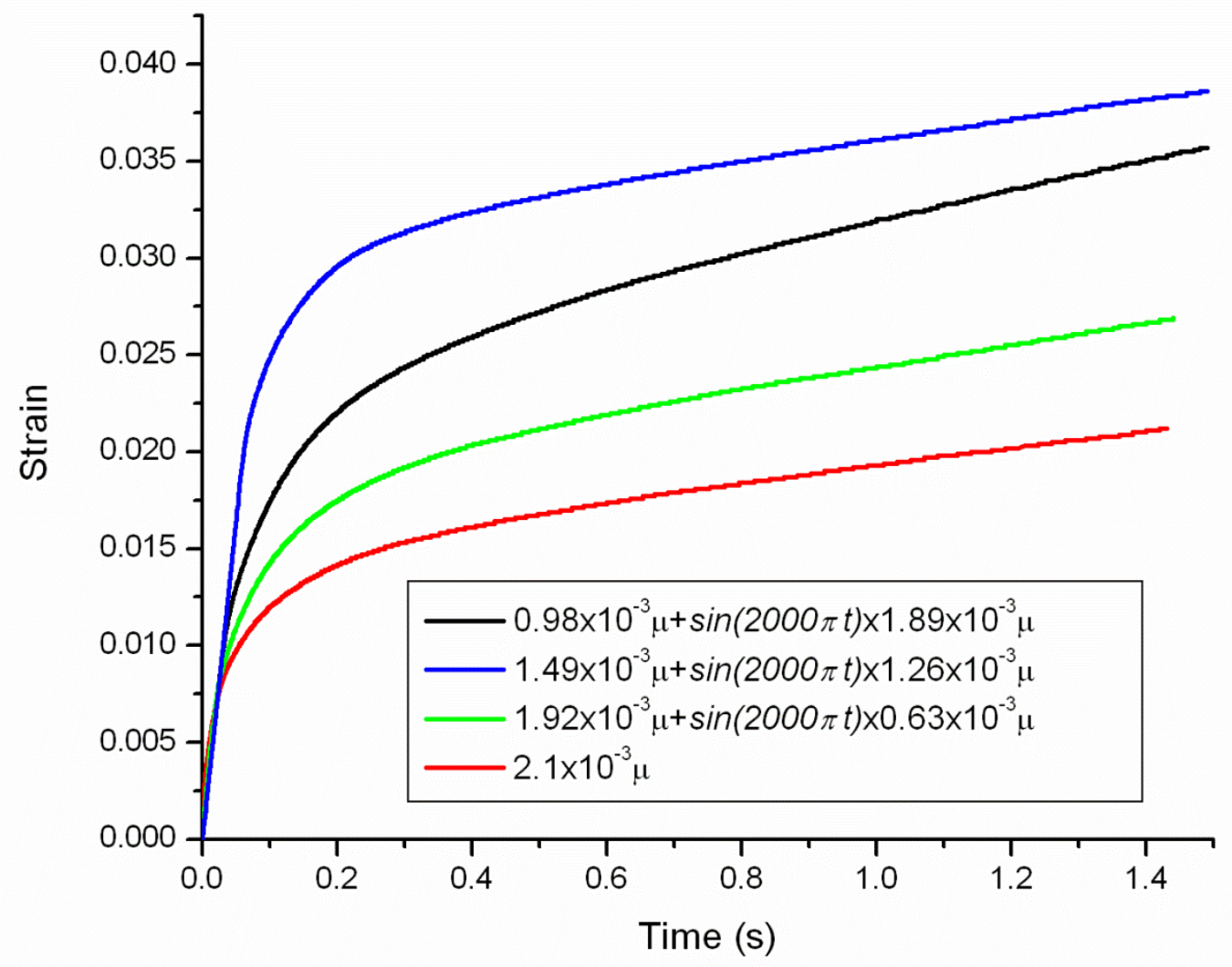

Figure 4 - Strain response of materials under different loading conditions. 


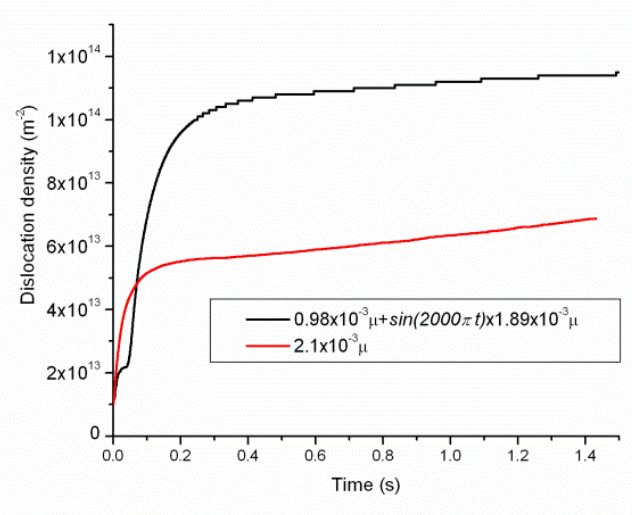

(a)

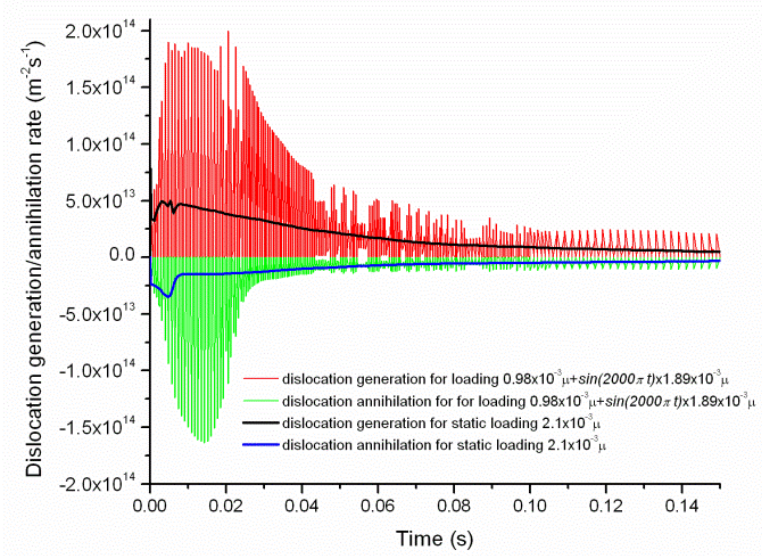

(b)

Figure 5 - (a) Overall dislocation density evolution during the simulations. (b) The dislocation generation and annihilation rate during the first $0.15 \mathrm{~s}$. 

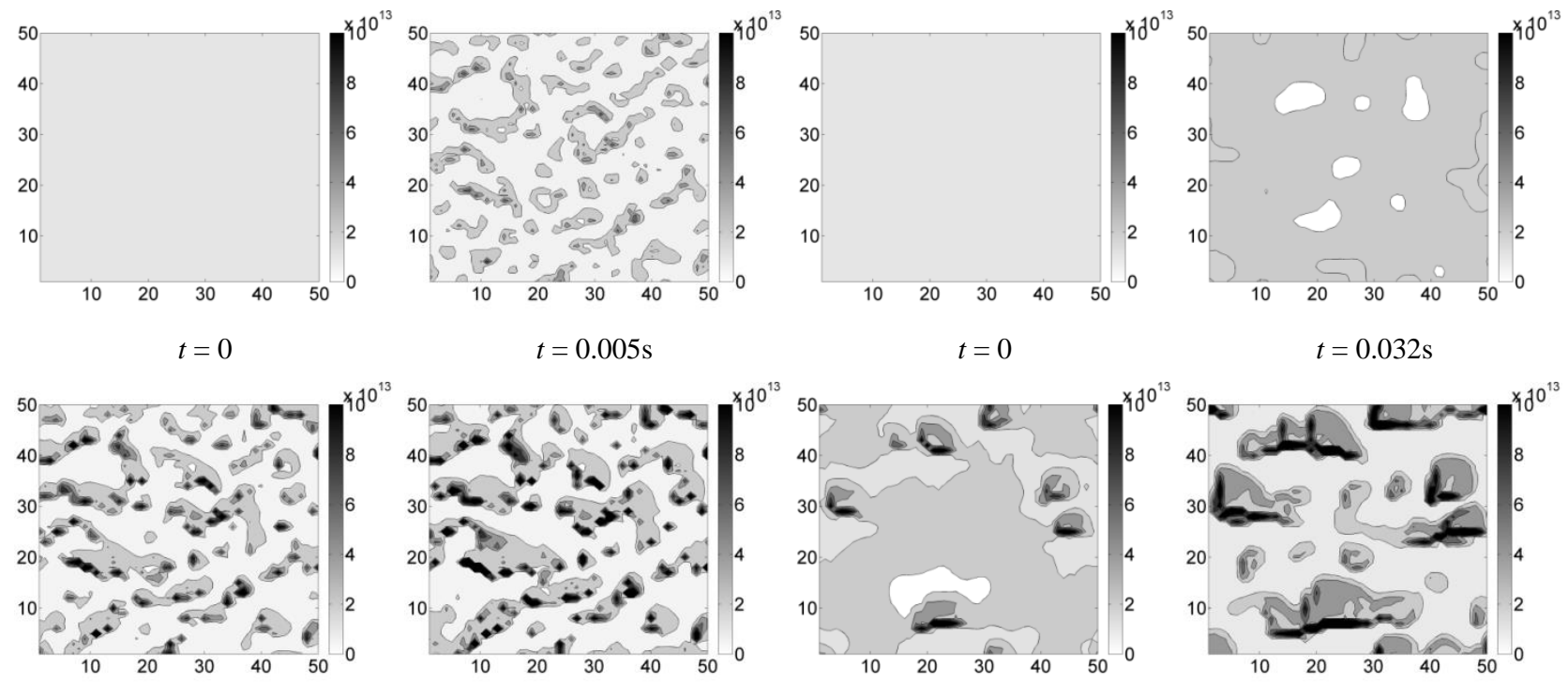

$t=0.009 \mathrm{~s}$

$t=0.014 \mathrm{~s}$

$$
t=0.045 \mathrm{~s}
$$

$t=0.054 \mathrm{~s}$
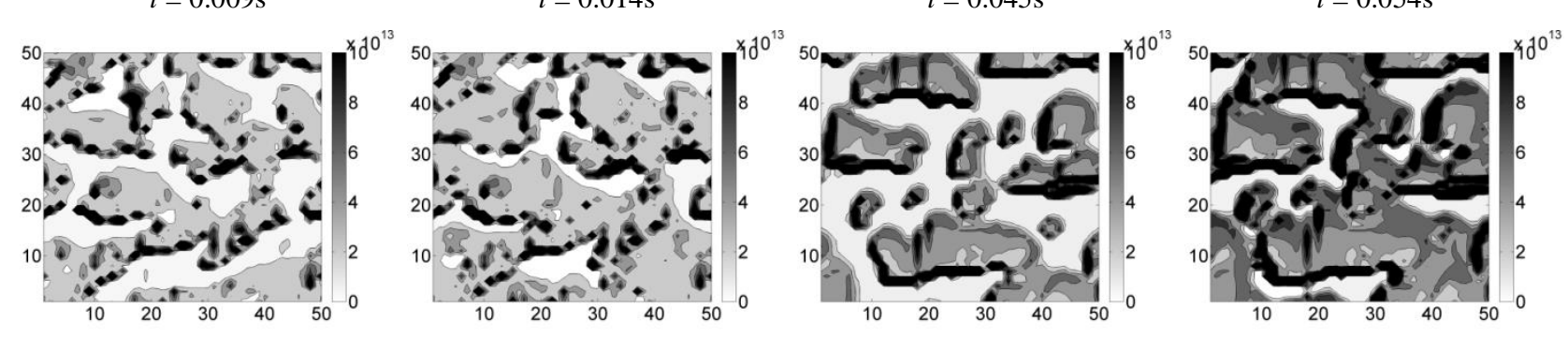

$t=0.032 \mathrm{~s}$

$t=0.072 \mathrm{~s}$

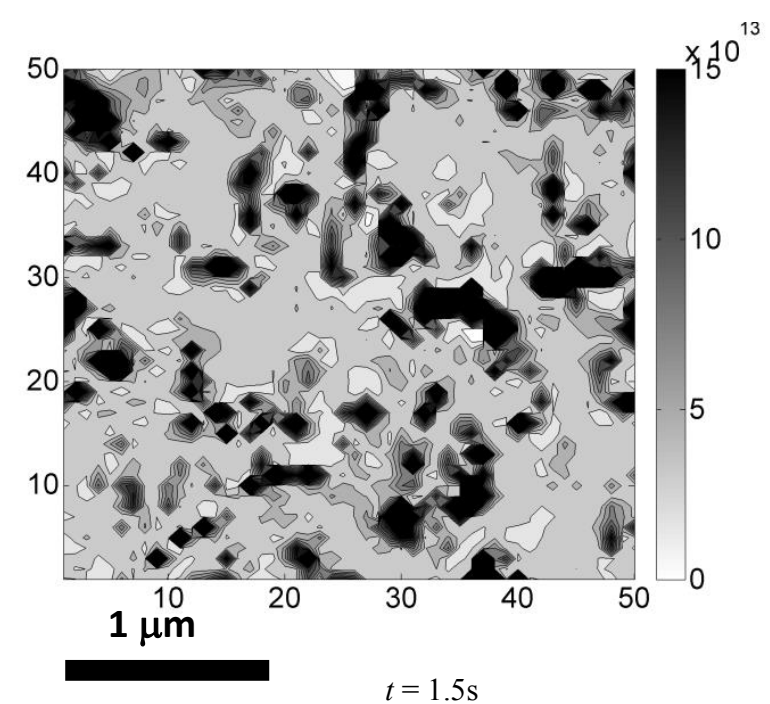

(a)

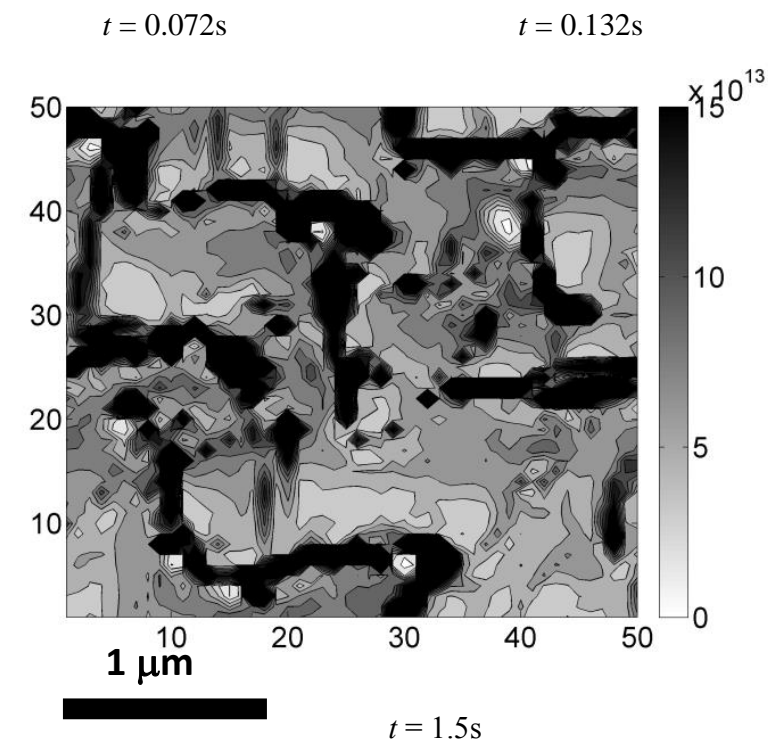

(b)

Figure 6 - Dislocation density $\left(\mathrm{m}^{-2}\right)$ evolution at different time points for (a) static loading of $2.1 \times 10^{-3} \mu$, (b) oscillatory loading of $0.98 \times 10^{-3} \mu+\sin (2000 \pi t) \times 1.89 \times 10^{-3} \mu$. Time is specified under each snapshot. 


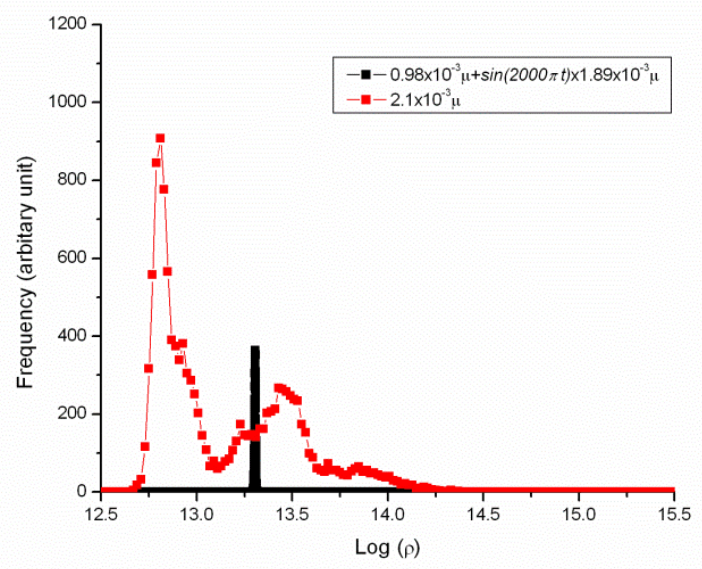

(a)

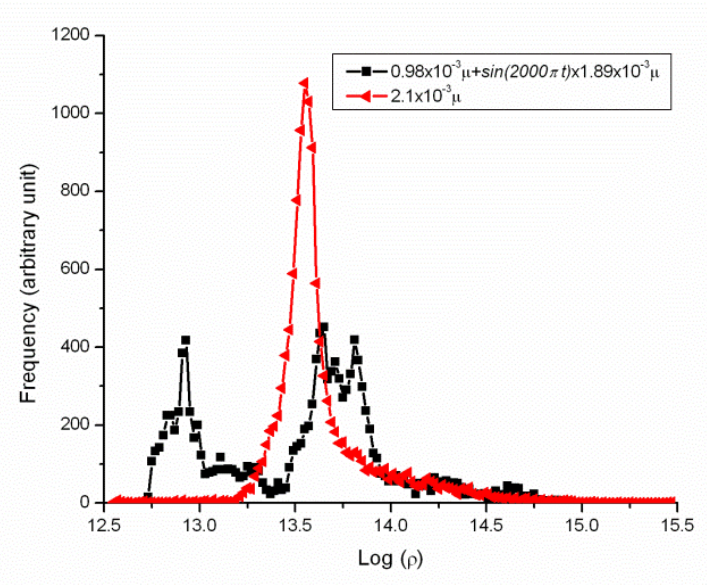

(c)

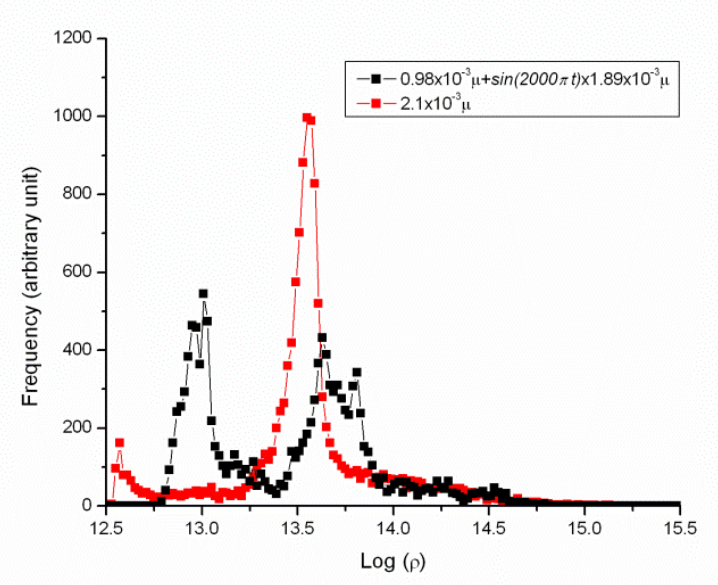

(b)

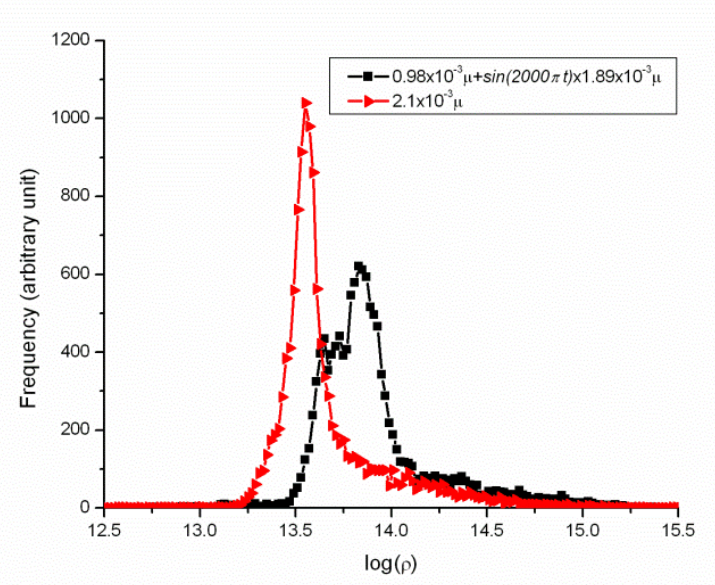

(d)

Figure 7 - Probability density functions for dislocation densities when the total dislocation densities under the two different loading conditions are both at (a) $2 \times 10^{13} \mathrm{~m}^{-2}$, (b) $5 \times$ $10^{13} \mathrm{~m}^{-2}$, and (c) $6 \times 10^{13} \mathrm{~m}^{-2}$. (d) shows the distribution at the end of the simulations. The abscissa is the $\log _{10}$ value of the total dislocation density, and the vertical scale denotes the occurrence frequency of a particular density in the simulation block. 

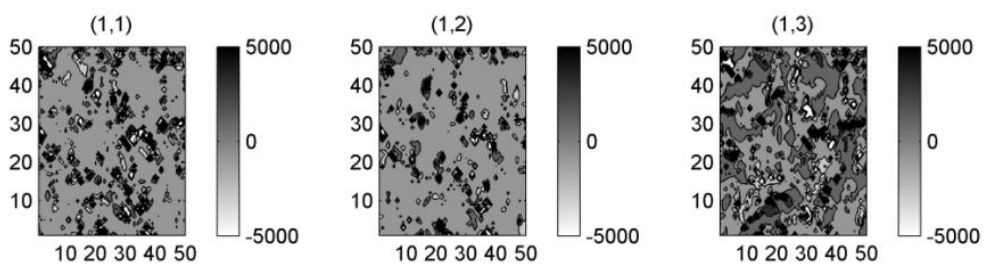

$(2,1)$
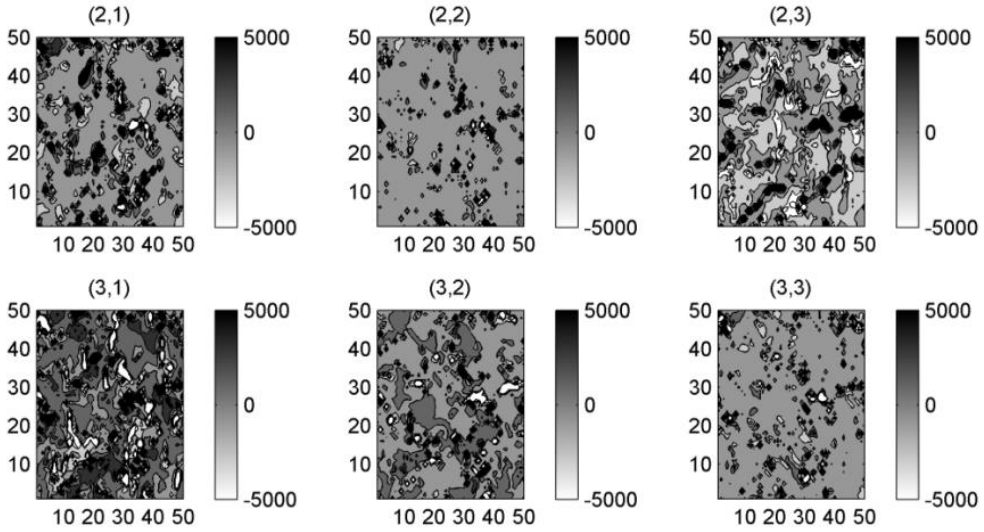

(a)
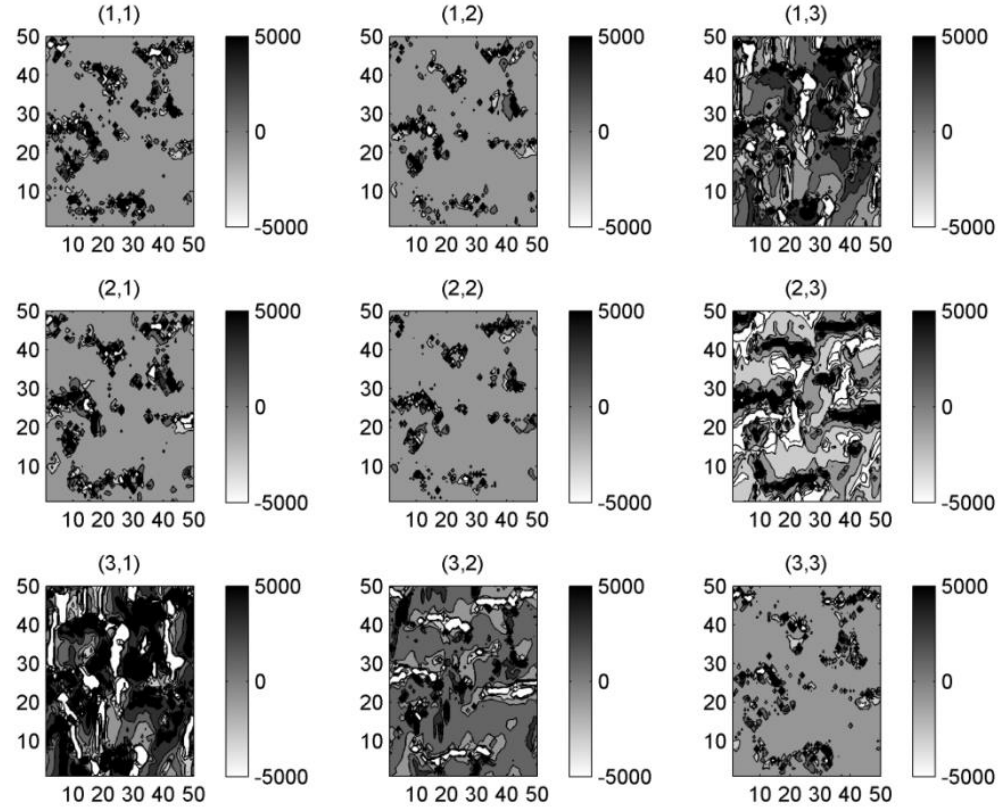

(b)

Figure 8 - Nye's tensor plot at the end of the simulations, in units of $\mathrm{m}^{-1}$. The label $(i, j)$ denotes the $A_{i j}$ component of the tensor. (a) Static loading of $2.1 \times 10^{-3} \mu$ only, (b) oscillatory loading of $0.98 \times 10^{-3} \mu+\sin (2000 \pi t) \times 1.89 \times 10^{-3} \mu$. 


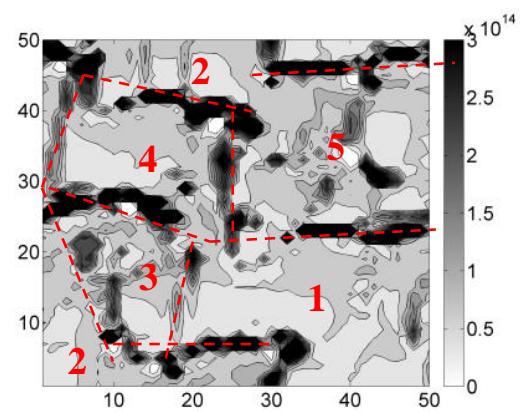

(a) $1.1 \times 10^{14} \mathrm{~m}^{-2}$

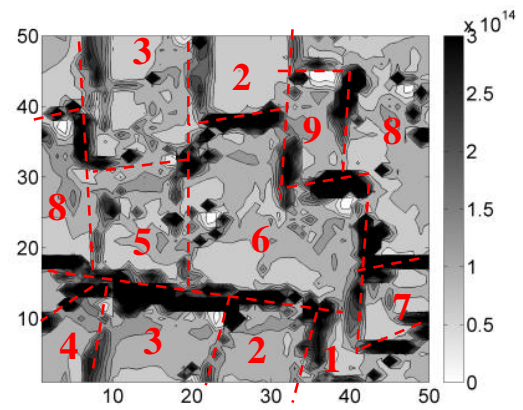

(b) $1.5 \times 10^{14} \mathrm{~m}^{-2}$

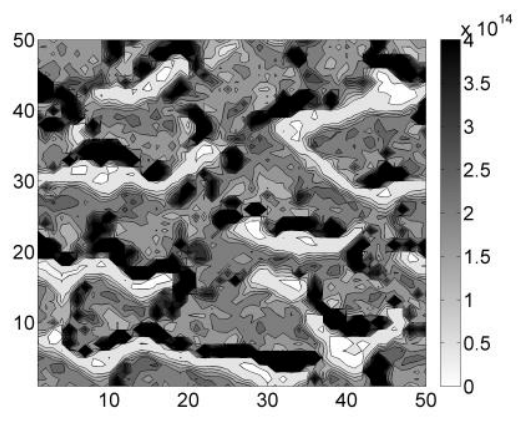

(c) $2.1 \times 10^{14} \mathrm{~m}^{-2}$

Figure 9 - Dislocation density distribution for specimens with different initial densities at strain $=3 \%$. The current total dislocation density is specified under each snapshot. (a) Specimen with initial density $10^{13} \mathrm{~m}^{-2}$ under oscillatory loading of $0.98 \times 10^{-3} \mu+\sin (2000 \pi t) \times 1.89 \times$ $10^{-3} \mu$. (b) Specimen with initial density $4 \times 10^{13} \mathrm{~m}^{-2}$ under oscillatory loading of $1.25 \times$ $10^{-3} \mu+\sin (2000 \pi t) \times 2.41 \times 10^{-3} \mu$. (c) Specimen with initial density $9 \times 10^{13} \mathrm{~m}^{-2}$ under oscillatory loading of $1.69 \times 10^{-3} \mu+\sin (2000 \pi t) \times 3.26 \times 10^{-3} \mu$. Red dash lines indicate the position of cell walls, and different cells are numbered. 


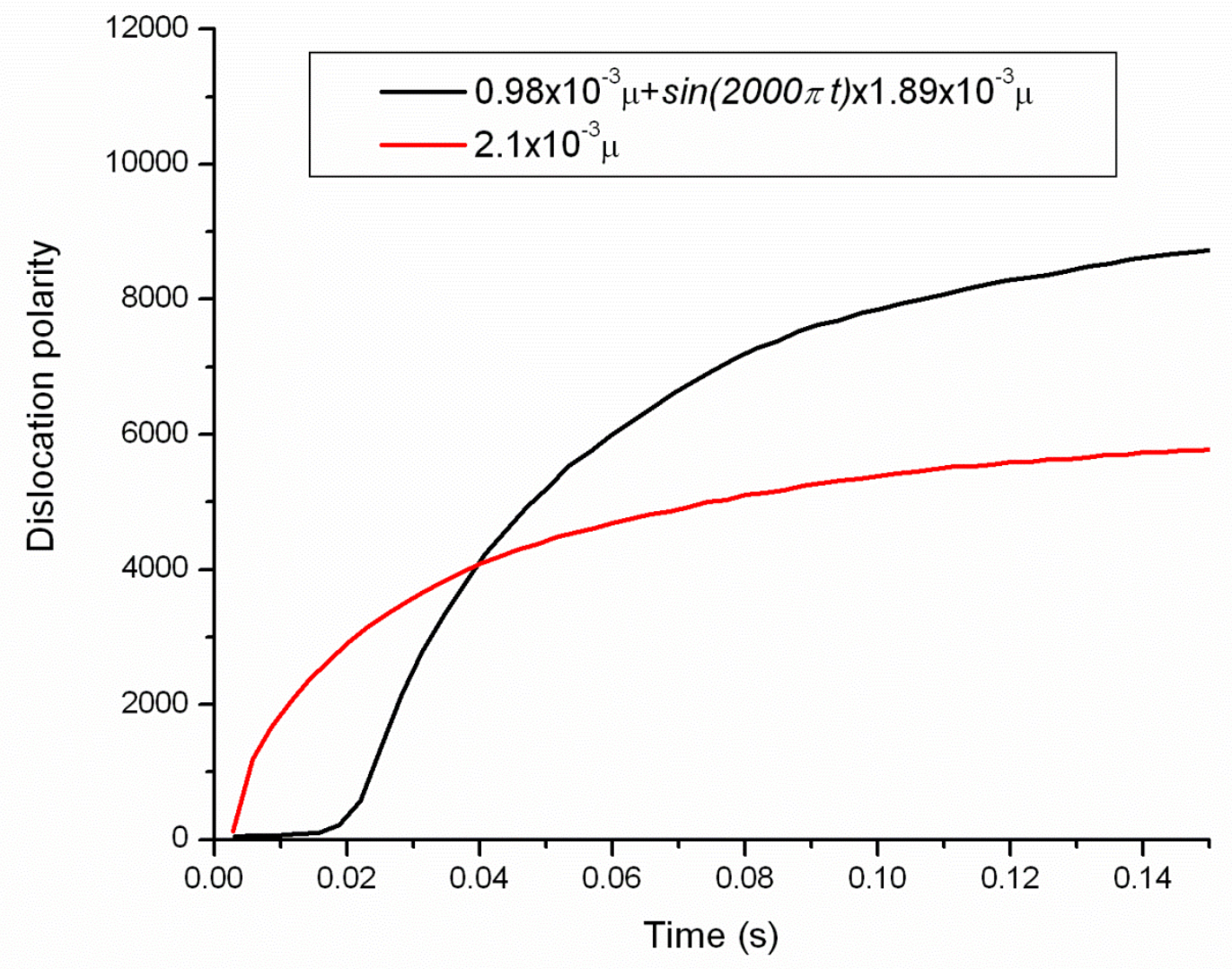

Figure 10 - Dislocation polarity evolution for the two cases in simulations. 


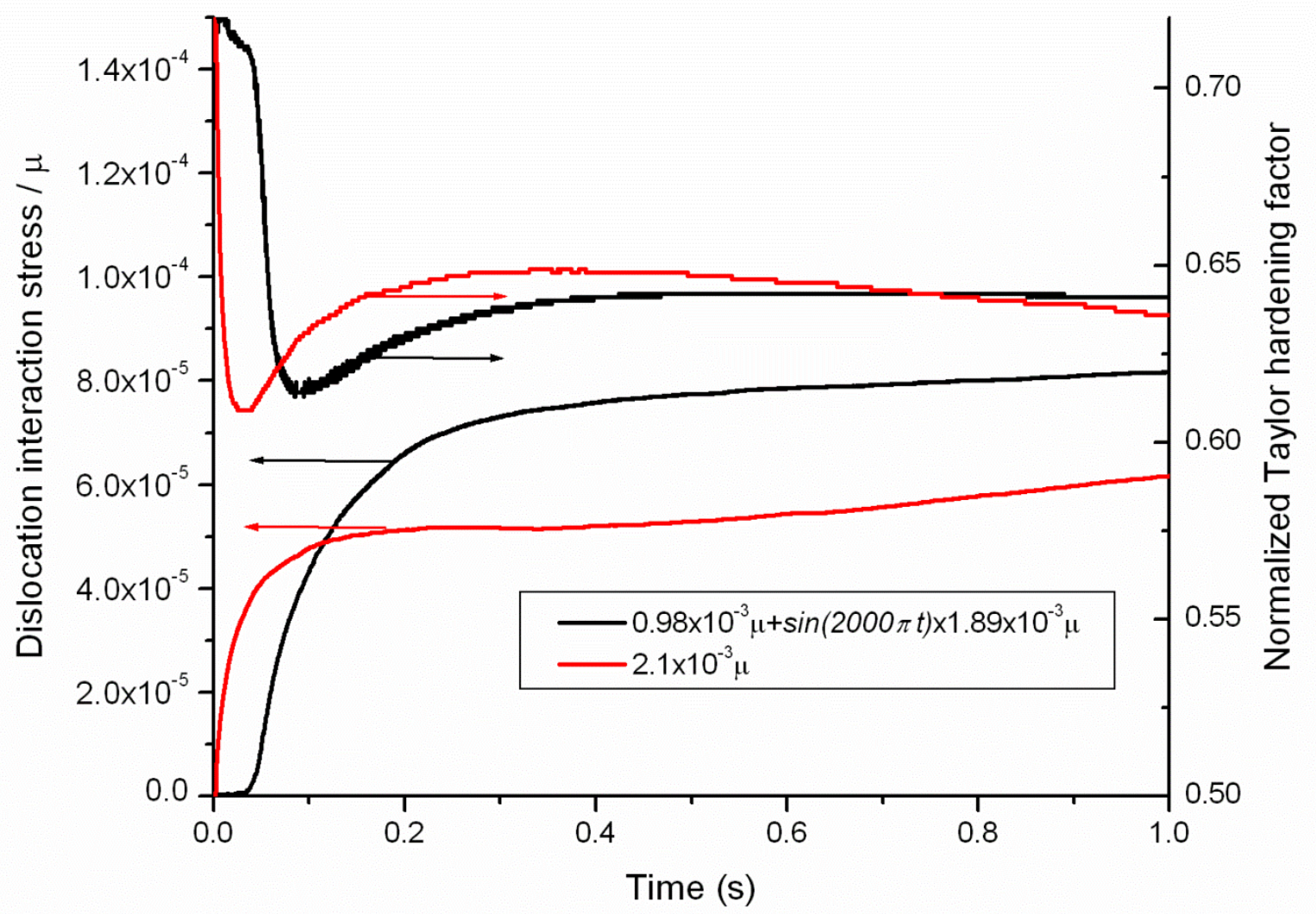

Figure 11 - The evolution of elastic interaction stress between dislocations $\overline{\left|\tau_{\alpha}^{\text {int }}\right|}$, and normalized Taylor hardening resistance $\overline{\mid \tau_{\alpha}^{\text {Taylor }}} \mid / \sqrt{\rho^{\text {total }}}$ for specimens under two different loading conditions. 


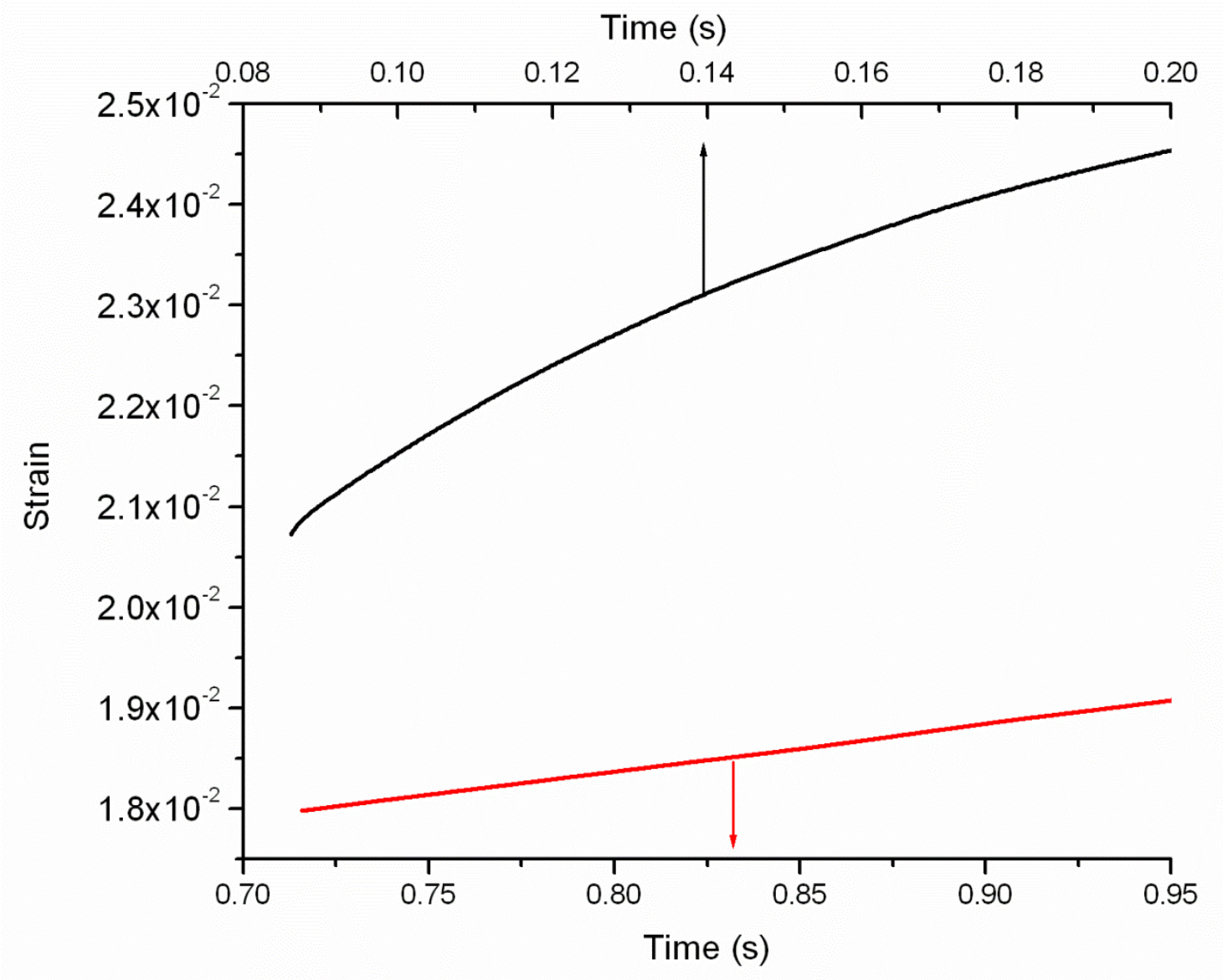

Figure 12 - The strain response of two specimens under the static loading of $2.1 \times 10^{-3} \mu$. Black curve: the specimen was pretreated with oscillatory loading of $0.98 \times 10^{-3} \mu+\sin (2000 \pi t) \times$ $1.89 \times 10^{-3} \mu$ for $0.086 \mathrm{~s}$ until the dislocation density reached $6 \times 10^{13} \mathrm{~m}^{-2}$. Red curve: the specimen was pretreated with static loading of $2.1 \times 10^{-3} \mu$ only, until the dislocation density reached $6 \times 10^{13} \mathrm{~m}^{-2}$. 